\title{
Improved Matching of Waveguide Focal Plane Arrays Using Patch Array Covers as Compared to Conventional Dielectric Sheets
}

\author{
Malcolm Ng Mou Kehn, Member, IEEE, and Lotfollah Shafai, Life Fellow, IEEE
}

\begin{abstract}
This paper presents the advantages of microstrip patch array covers as matching layers for waveguide arrays, as compared with the use of conventional dielectric sheets. It will be demonstrated that both types of covers can be tuned to improve the transmission levels of uncovered arrays at either low frequencies or over a very wide band. However, at low frequencies where the radiation performance tends to be poor due to strong mutual coupling between closely separated elements, the patch array cover offers itself as a lower profile and lighter weight matching structure. This makes it highly advantageous for use in space applications and satellite communications. In addition, it is also able to provide wider matching bandwidths than dielectric sheets. Here, we present a rigorous analysis of such a patch array printed on a dielectric slab and placed over an array of sidewall loaded hard rectangular waveguides. Due to the desirable properties of its aperture field, this type of element serves as an ideal reference for conveying the properties of the two matching layers studied here. Also, to ensure the matching layer performance is independent of the array elements, optimized elements are used in all computations. The method of moments using spectral Green's functions for multilayer structures is employed. Two array operation scenarios are studied, which are the direct radiating phased arrays, and focal plane arrays (FPAs) as feeds for reflectors.
\end{abstract}

Index Terms-Array matching, focal plane array (FPA) feed, hard waveguide array, microstrip patch array, sheath-covered waveguide array.

\section{INTRODUCTION}

A RRAY antennas may be used as either direct radiating phased arrays (DRPA) in which beams are formed directly by the array aperture, or multibeam cluster feeds for reflector antennas [1]-[5]. Recently, there has also been increased attention on wideband dense focal plane array (FPA) feeds [6], [7] comprising elements with diameters up to half a wavelength.

A major challenge in DRPAs is the impedance matching. As the element impedance varies with scan, excessive scanning incurs mismatch (reflection) losses. There had been earlier studies

Manuscript received September 09, 2008; revised April 21, 2009. First published July 28, 2009; current version published October 07, 2009.

M. Ng Mou Kehn was with the Department of Electrical and Computer Engineering, University of Manitoba, Winnipeg, MB R3T 5V Canada. He is now with the Department of Electrical Engineering, National Chiao Tung University, Hsinchu 300, Taiwan

L. Shafai is with the Department of Electrical and Computer Engineering, University of Manitoba, Winnipeg, MB R3T 5V Canada (e-mail: shafai@ee. umanitoba.ca).

Color versions of one or more of the figures in this paper are available online at http://ieeexplore.ieee.org.

Digital Object Identifier 10.1109/TAP.2009.2028624 on ways of achieving widescan matching, e.g., by using interconnecting circuits [8], [9]. But still, to maintain widescan match over a broad frequency range remains difficult, if not unresolved. For open-ended waveguide arrays, other means of matching include the insertion of dielectric sections within the waveguides [10], [11] or placement of dielectric sheath layers over the array aperture, to serve as impedance tuners [12]. Recently, there has been a reported work on a new body of revolution (BOR) type of dual-polarized broad band array element that is able to attain broad scan range over a wide impedance bandwidth [13].

For the case of dense FPAs, being a newer type of array than conventional DRPAs, even fewer explorations into the matching issue have been done. In fact, there are no known prior efforts devoted to the matching of FPAs. Unlike DRPAs, the elements of an FPA are arbitrarily and independently excited. This makes it very difficult to match every element over a wide frequency range. This poses the main obstacle to the matching of FPAs.

In [14] and [15], it was shown and stressed that an important factor affecting the overall performance of an FPA-fed reflector is the coupling efficiency of the array feed. This figure of merit characterizes the severity of mutual coupling losses among the array elements. It was found that the coupling effects in FPAs strengthen with reduced inter-element separation. As such, the coupling efficiency tends to be poor at low frequencies, due to the close electrical proximity between elements. This efficiency thus constitutes a major degradation factor of the total efficiency of an FPA-fed reflector system, especially at low frequencies. Therefore, the pertinence of array matching through the improvement of the coupling efficiency cannot be overemphasized. This also motivates the particular quest for a means of array matching within the low frequency bands, for both DRPAs and FPAs.

A traditional method of array matching is the use of a dielectric sheet placed over the array [12] as an impedance tuner. It however suffers from likewise age-old problems such as weight, profile, and bandwidth issues, especially at low frequencies where the radiation deficiency is the most severe. Here, a new type of matching structure is proposed and studied, which is a periodic array of metallic patch elements printed over a thin low permittivity dielectric layer. The purpose of this work is to present the matching capabilities of these two kinds of array covers and compare their performances. In the process of doing so, we will demonstrate that both forms of match tuners are able to achieve either low-frequency or wideband matching. However, it will be shown that the patch array cover is more 


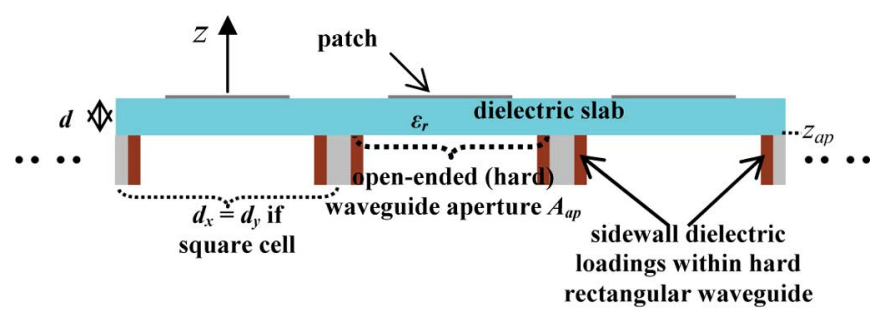

(a)

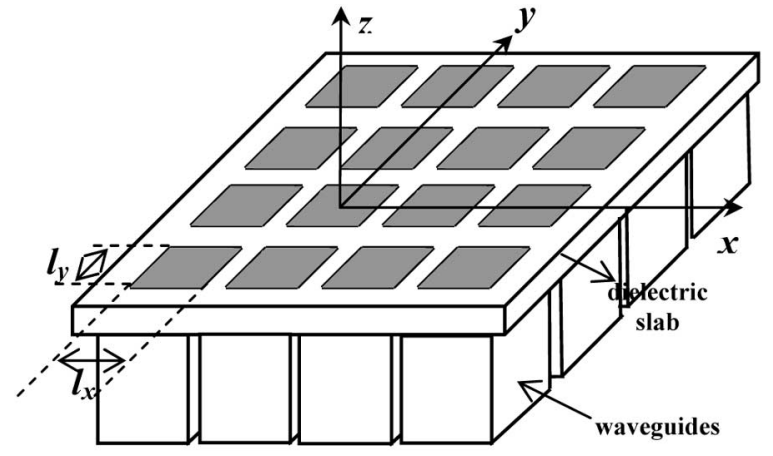

(b)

Fig. 1. (a) Side view of a waveguide array covered by a patch array layer comprising an array of metallic patches etched on a dielectric slab. The rectangular waveguides are sidewall loaded by dielectric slabs as shown, serving as hard waveguides. (b) Perspective view of an example RPA layer composed of rectangular patches, covering an array of rectangular waveguides.

advantageous in terms of the difficulties faced by conventional sheets at low frequencies, as mentioned above. In addition, it will be low profile and low cost, as its supporting dielectric layer is only for mechanical support, and need not be thick or heavy, a particularly important advantage for mobile and space applications.

The configuration of the waveguide array covered by a rectangular patch array (RPA) is depicted in Fig. 1, illustrating an example case of sidewall loaded hard rectangular waveguides (HRW) studied in [10] as DRPA elements, and later on in [14] as FPA elements. Such HRW array elements will be reused here for the present study. This type of element serves as an ideal reference due to the desirable properties of its aperture field. The waveguide array configurations and operation scenarios (DRPA and FPA) are pictorially summarized in Fig. 2.

Full mutual coupling analyses are performed on the arrays studied here. The mutual coupling coefficients under the so-called embedded element scenario (in which only one element is excited while the surrounding ones are still present but unexcited) are obtained by integrating the DRPA active reflection coefficients (for numerous phasings) over the Brillouin zone. Finite arrays of any excitation may then be treated by an element-by-element approach entailing the superposed sum of mutual coupling losses in all considered elements. More on this will be explained later, but for now, it is asserted early that the DRPA solution only serves as an auxiliary step towards the treatment of finitely excited arrays. It is also appropriate at this juncture to declare that the "finite array" investigated here and referred to throughout this paper is defined as a physically infinite array with a finite number of excited elements. The other elements outside the excited portion are passive, i.e.,

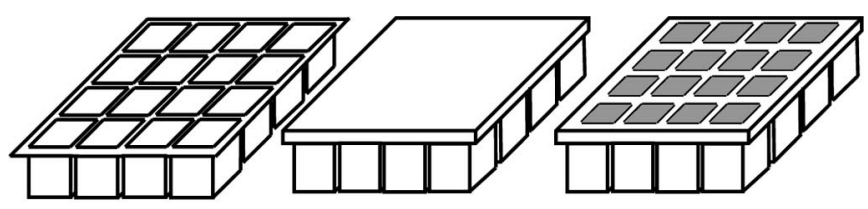

(i) uncovered

(ii) sheath-covered

(iii) RPA-covered

(a)

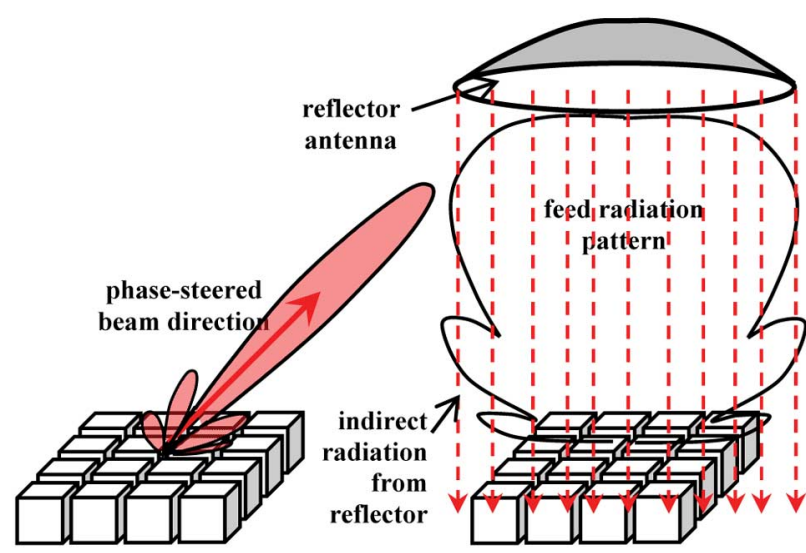

(i) DRPA

(ii) FPA feed for reflector (grossly out of scale)

(b)

Fig. 2. Waveguide array structures and operation scenarios: (a) Three waveguide array configurations investigated: (i) uncovered, (ii) plain sheet covered, and (iii) RPA-covered. (b) Two operation scenarios: (i) DRPA, and (ii) FPA feed for reflector antennas: indirect radiation via ray collimation by the reflector.

nonexcited [16]. Such a finite array is only hypothetical, as a true finite array would contain only a finite number of physically existent elements. Nonetheless, the properties of a true finite array can be in most cases well approximated to those of a finite array as defined above. The reason for this is that the contribution of the passive elements to the array radiation is most often negligible [16].

As a final note before proceeding, it is stated that the patch array covers studied here are aligned with the waveguide openings underneath. There is, however, a large potential for further explorations through the study of offset patches relative to the midpoint of the waveguide apertures.

\section{INFINITE PhaSED ARRAY SOlUtion AS AuXILIARY FOR THE TREATMENT OF ARBITRARILY EXCITED FINITE ARRAY}

This section analyzes the waveguide-aperture coupled patch array printed over a dielectric slab, operated as a DRPA. The solution of this scenario serves as an auxiliary stage for the subsequent treatment of arbitrarily excited finite arrays. This will be described more in the next section.

\section{A. Expansion of Electric and Magnetic Currents over PEC Patch and Waveguide Aperture Into Basis Functions}

Consider the RPA-covered waveguide array shown in Fig. 1, with its surface parallel to the $x-y$ plane, and periods along $x$ and $y$ being $d_{x}$ and $d_{y}$, respectively. In the present approach, the electric current on the perfect electric conducting (PEC) patch is expanded out into subsectional vectorial Rao-Wilton-Glisson (RWG) basis functions [17]. The triangular domains of such 
basis functions are thus able to treat arbitrarily shaped metallizations. In spectral $\left(k_{x}, k_{y}\right)$ domain, evaluated at discrete coordinates defined in (3a) and (3b), this patch electric current is written as

$$
\begin{aligned}
& \stackrel{\approx}{\vec{J}}^{\text {patch }}\left(k_{x m}, k_{y n}\right)=\sum_{p=1}^{N_{\text {patch }}} a_{p}^{\text {patch }} \stackrel{\approx_{\vec{f}}^{\text {RWG }}}{p}\left(k_{x m}, k_{y n}\right)
\end{aligned}
$$

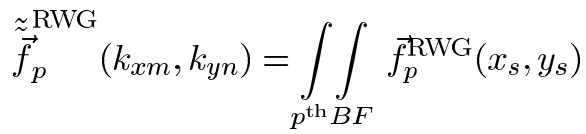

$$
\begin{aligned}
& \times e^{j\left(k_{x m} x_{s}+k_{y n} y_{s}\right)} d x_{s} d y_{s}
\end{aligned}
$$

where the latter is the spectral $p$ th RWG basis function, which is weighted by amplitude coefficient $a_{p}^{\text {patch }}$ in (1a). The $s$ subscript denotes source coordinates and $N_{\text {patch }}$ is the number of basis functions. The domain of integration spans over the triangular domain of the $p$ th RWG basis function, as indicated. The double tilde represents spectral domain of the associated quantities. This applies also to the subsequent equations.

The spectral PEC equivalent magnetic current source over the radiating aperture at $z_{\text {ap }}$ just outside the aperture is given by

$$
\begin{aligned}
\tilde{\vec{M}}^{\text {apert }}\left(k_{x m}, k_{y n}\right)= & \sum_{p q r=1}^{N_{\text {guide }}} A_{p q r} \tilde{\overrightarrow{\vec{g}}}_{p q r}\left(k_{x m}, k_{y n}\right) \\
\tilde{\overrightarrow{\vec{g}}}_{p q r}\left(k_{x m}, k_{y n}\right)= & \iint_{A_{p}}\left(\hat{x} e_{y_{p q r}}-\hat{y} e_{x_{p q r}}\right) \\
& \times e^{j\left(k_{x m} x_{s}+k_{y n} y_{s}\right)} d x_{s} d y_{s}
\end{aligned}
$$

where $N_{\text {guide }}$ is the number of waveguide basis functions, $p q r$ is the triple mode index typical of waveguide modes, and $A_{p q r}$ is the expansion coefficient of the $(p q r)$ th mode. The symbol $e$ (and later, $h$ as well) is the electric (and magnetic) modal field function of the waveguide. Its subscript ( $x$ or $y$ ) denotes the field component. These modal functions are not explicitly presented here, but may be acquired from [10]. The domain of integration in (2a) spans over the waveguide aperture $A_{p}$, as indicated. The discrete spectral coordinates occurring in the preceding equations are given by

$$
\begin{aligned}
k_{x m} & =k_{0} \sin \theta_{0} \cos \phi_{0}+2 m \pi / d_{x} \\
k_{y n} & =k_{0} \sin \theta_{0} \sin \phi_{0}+2 n \pi / d_{y}
\end{aligned}
$$

where $m$ and $n$ are integers, $\theta_{0}$ and $\varphi_{0}$ are angular coordinates defining the direction of the dominant Floquet modal beam, and $k_{0}$ is the usual freespace wavenumber. Under phased array scenario, $\left(\theta_{0}, \varphi_{0}\right)$ defines the steered main beam direction.

\section{B. Spectral Fields Radiated by Spectral Basis Currents}

The spectral basis currents of (1b) and (2b) are subsequently employed as the driving excitation for the bare multilayer structure without the patches. The spectral fields radiated by these spectral basis currents into the $i$ th layer ( $i$ may denote 0 for the freespace above the slab, or $d$ for the slab) are obtained from the numerical spectral Green's function of the unloaded multilayer structure using the G1DMULT core routine [18], [19], i.e.,

$$
\tilde{\tilde{F}}_{w(i)}^{\kappa_{s_{b}}}=\tilde{\widetilde{G}}_{F_{w}(i)}^{K_{s}} \cdot \tilde{\tilde{\kappa}}_{s_{b}}
$$

where the $w$ subscript may denote either $x$ or $y$ component of the spectral $F$-field, this $F$ being either $E$ or $H$, and the spectral dyadic Green's function $G$ is characterized by superscript $K_{s}$ signifying the type ( $K$ is $J$ or $M$ ) and component ( $s$ is $x$ or $y$ ) of the excitation source (the subscript of $G$ indicates the field type and component). The driving spectral basis current is labeled by $\kappa$, which may represent either $f^{\text {RWG }}$ or $g$. The sub-subscript $b$ denotes either $p$ or $p q r$ (the indices of the basis function) depending whether $\kappa$ is $f^{\mathrm{RWG}}$ or $g$, respectively. The complete details of this numerical spectral Green's function are too voluminous and thus are not provided here.

\section{Spatial Fields Radiated by Entire Array of Patch and Aperture Currents}

Due to the periodicity along $x$ and $y$ with periods $d_{x}$ and $d_{y}$, the inverse transform from spectral $k_{x}$ to spatial $x$ domain only requires a summation over discrete spectral components defined in (3a) and (3b). Hence, the inverse transform of the spectral field of (4) is written as

$$
F_{w(i)}^{\kappa_{s_{b}}}=\frac{1}{d_{x} d_{y}} \sum_{m, n} \tilde{\tilde{F}}_{w(i)}^{\kappa_{s_{b}}} e^{-j\left(k_{x m} x+k_{y n} y\right)} .
$$

The summation runs through integers of $m$ and $n$. Combining the $x$ and $y$ field components ( $w$ subscript)

$$
\vec{F}_{t(i)}^{\kappa_{s_{b}}}=\hat{x} F_{x(i)}^{\kappa_{s_{b}}}+\hat{y} F_{y(i)}^{\kappa_{s_{b}}}
$$

where the " $t$ " signifies "transverse" $x y$ components. This (6) then constitutes the transverse component of the spatial-domain field radiated by the $s$-directed spatial $b$ th basis current $\kappa_{s_{b}}(x, y)$

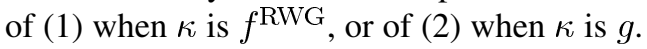

\section{Galerkin Weighting of Boundary Conditions}

There are two boundary conditions to be satisfied, one on the PEC patch, and the other on the waveguide aperture. For the patch, the boundary condition requires the component of the total electric field tangential to the PEC patches to vanish on the patch surface. The other boundary condition requires the continuity of the tangential magnetic field across the waveguide aperture.

Upon performing Galerkin weighting, a total number of equations that equals the number of unknown coefficients of the basis functions expanding the patch and aperture currents are generated. This system of equations can be cast into a matrix equation

$$
[\bar{Z}][\bar{I}]=[\bar{V}]
$$

where

$$
\begin{array}{ll}
{[\bar{Z}]=\left\{\begin{array}{ll}
{\left[\bar{Z}_{11}\right]} & {\left[\bar{Z}_{12}\right]} \\
{\left[\bar{Z}_{21}\right]} & {\left[\bar{Z}_{22}\right]}
\end{array}\right\}} \\
{[\bar{V}]=\left\{\begin{array}{c}
{[O]_{N_{\text {patch }} \times 1}} \\
{\left[\bar{V}_{\text {apert }}\right]_{N_{\text {guide }} \times 1}}
\end{array}\right\} \quad[\bar{I}]=\left\{\begin{array}{l}
{\left[a_{p}^{\text {patch }}\right]_{N_{\text {patch }} \times 1}} \\
{\left[A_{p q r}\right]_{N_{\text {guide }} \times 1}}
\end{array}\right\}}
\end{array}
$$


where $[O]$ is an $N_{\text {patch }} \times 1$ column vector of zeros. The $\left[a_{p}^{\text {patch }}\right]$ and $\left[A_{p q r}\right]$ column vectors contain the unknown amplitude coefficients of the basis functions expanding the currents, which are to be solved for.

The $(q, p)$ th element (row index, column index) of the square matrix $\left[\bar{Z}_{11}\right]$ in (8), having a size $N_{\text {patch }} \times N_{\text {patch }}$, is written as

$$
Z_{11_{q, p}}=\iint_{q^{\text {th }}}\left(\vec{E}_{t(0)}^{J_{x_{p}}^{\text {patch }}}+\vec{E}_{t(0)}^{J_{y_{p}}^{\text {patch }}}\right) \cdot \vec{f}_{q}^{\mathrm{RWG}} d x d y
$$

where the vector $E$-field quantities in the integrand are obtained from (6). The surface integration spans over the triangular domain of the $q$ th RWG basis (or now, weighting) function.

In a similar fashion, the $(q, p q r)$ th element of the matrix $\left[\bar{Z}_{12}\right]$ of size $N_{\text {patch }} \times N_{\text {guide }}$ is

$$
Z_{12_{q, p q r}}=\iint_{q^{\text {th }}}\left(\vec{E}_{t(0)}^{M_{x p q r}^{\mathrm{apert}}}+\vec{E}_{t(0)}^{M_{y p q r}^{\mathrm{apert}}}\right) \cdot \vec{f}_{q}^{\mathrm{RWG}} d x d y
$$

where the integrand fields are from (6) again. The $q$ represents the index of the weighting function $\vec{f}_{q}^{\mathrm{RWG}}(x, y)$, which runs through the same indices as those of $p$, being the index of the RWG basis functions. Proceeding to the other submatrices, the $(r s t, p)$ th element (row index, column index) of the matrix $\left[\bar{Z}_{21}\right]$ with size $N_{\text {guide }} \times N_{\text {patch }}$ is

$$
Z_{21_{r s t, p}}=\iint_{A_{\mathrm{ap}}} \vec{e}_{t_{\mathrm{rst}}} \times\left(\vec{H}_{t(d)}^{J_{x_{p}}^{\mathrm{patch}}}+\vec{H}_{t(d)}^{J_{y_{p}}^{\mathrm{patch}}}\right) \cdot \hat{z} d x d y
$$

where the $H$-fields in the integrand are again acquired from (6). The $r s t$ signifies the index of the weighting function, being also the waveguide modal basis function (electric modal field) expanding the aperture magnetic current as expressed in (2). Finally, the $(r s t, p q r)$ th element of the square matrix $\left[\bar{Z}_{22}\right]$ with size $N_{\text {guide }} \times N_{\text {guide }}$ is

$$
\begin{aligned}
& Z_{22_{r s t, p q r}} \\
& \quad=\iint_{A_{\mathrm{ap}}} \vec{e}_{t_{\mathrm{rst}}}\left(\vec{H}_{t(d)}^{M_{x q r}^{\text {patch }}}+\vec{H}_{t(d)}^{M_{y q r}^{\text {patch }}}+\sum_{u v w=1}^{N_{\text {guide }}} C_{p q r, u v w} \vec{h}_{t_{p q r}}\right) \\
& \quad \cdot \hat{z} d x d y
\end{aligned}
$$

whereby

$$
C_{p q r, u v w}=\iint_{A_{p}}\left(e_{x_{p q r}} h_{y_{\mathrm{uvw}}}-e_{y_{p q r}} h_{x_{\mathrm{uvw}}}\right) d x d y
$$

is the intermodal coupling coefficient $C_{p q r, u v w}$ for the coupling between the ( $p q r)$ th and (uvw)th modes within the same waveguide with cross-sectional area $A_{\mathrm{ap}}$.

The subvector $\left[\bar{V}_{\text {apert }}\right]$ in (9) is a column vector containing all zeros except the element at the node location pertaining to the incident mode index, i.e.,

$$
\left[\bar{V}_{\text {apert }}\right]_{N_{\text {guide }} \times 1}=\left[\begin{array}{lllll}
0 & \cdots & e^{-\gamma_{z_{\text {in }}}\left(z_{\text {ap }}-z_{\text {gen }}\right)} & \cdots & 0
\end{array}\right]^{T}
$$

where $T$ symbolizes the nonconjugate transpose. The $\gamma_{z}$ is the propagation constant of the incident mode traveling along the axial $z$-direction, and its subscript "in" represents the incident mode index. The quantity $z_{\text {gen }}$ is the source reference point of the generator inside each waveguide.

\section{ARbitrariLy EXCITED Finite ARRAY With FUlL Mutual COUPLing ANALYSIS VIA PHASED ARRAY AUXILIARY}

In the same manner as presented in [20], upon solving the phased array (DRPA) scenario as described in the preceding section, a finite RPA-covered HRW array excited by any arbitrary function can be treated. There are two ways of doing so: 1) mutual coupling analysis cum element-by-element approach entailing the summation of reflected and coupled power losses receded back into the array element generators, and 2) superposition of amplitude-scaled and phase-shifted embedded element patterns to obtain the finite array radiation pattern, from which the total radiated power can be calculated.

The former method is performed by first repeatedly solving this DRPA scenario for the active reflection coefficient over numerous phasings (steered beam directions). These DRPA reflection coefficients are then multiplied by associated exponential kernel functions (of Fourier transform, read further below) and integrated over the Brillouin zone to obtain the embedded element mutual coupling coefficients. This is based on the classical theorem stating that the active reflection coefficient of the infinite phased array and the coupling coefficient under the embedded element scenario constitute a Fourier transform pair [21]. There is one such embedded element coupling coefficient for each waveguide mode, i.e., the coupling from an excited mode of any one waveguide element to another generally different coupled mode of any other element. With these element coupling coefficients for various modes computed, any arbitrarily excited finite array (e.g., the FPA excited according to the Airy pattern, as studied here-see later) is solved via an element-by-element approach. This means that the power loss in any one array element is calculated by summing up the mutually coupled modal power losses contributed by all surrounding elements as well as the self-coupling loss, i.e., the reflection loss. This is then repeated for all elements considered in the finite array. Hence, the solution of the phased array scenario only serves as an auxiliary step for treating the finite array. Due to space constraint, the mathematical details of these theories and concepts cannot be presented here, but more information may be found in [20].

The validity of the mutual coupling approach just described can be verified by an alternative classical approach (the second method cited above), which has also been done in our work. This entails the superposition of embedded element patterns to obtain the radiation pattern of the arbitrarily excited finite array, whereby each embedded element pattern is weighted according to the amplitude excitation coefficient of its respective constituent element, together with an appropriate phase shift according to its location in the finite array. The total power loss in the finite array calculated using these two approaches will be equal, i.e., the total power loss in the finite array obtained via the element-by-element approach (summing up all mutual and self coupling power losses in all elements) is equal to the one acquired by subtracting the total radiated power carried by the superposed embedded element patterns (being the power pattern 

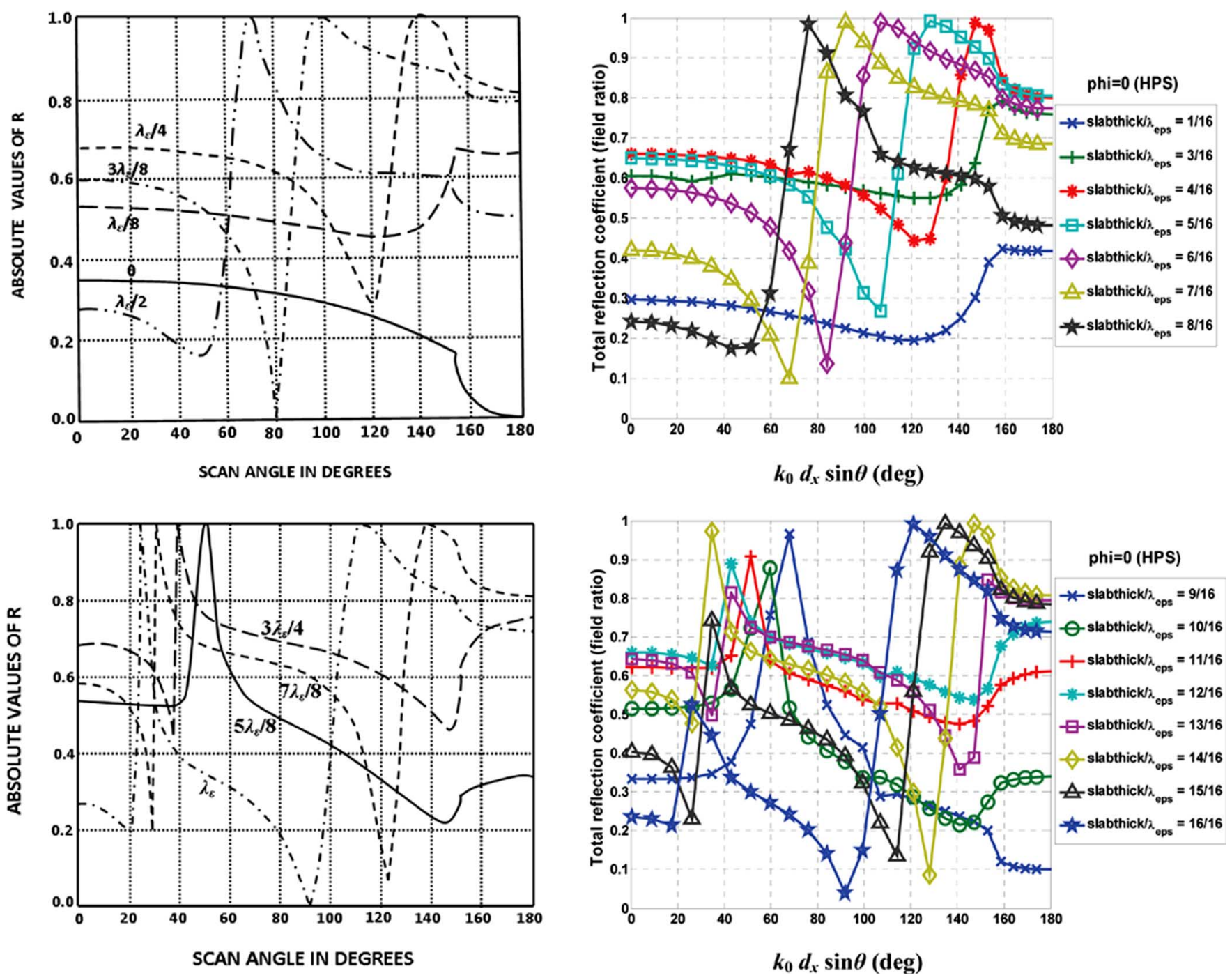

Fig. 3. Validation results of [21, Figs. 6.12 and 6.13] shown as left-hand side plots, reconstructed as the right-hand side plots generated by our code. Graphs of DRPA reflection coefficient versus electrical phasing angle $k_{0} d_{x} \sin \theta$ (where $d_{x}$ is the period along $x$ ) of a sheathed rectangular waveguide array, for $H$-plane scan and with slab thickness (normalized to $\lambda_{\varepsilon}=\lambda_{0} / \sqrt{ } \varepsilon$ ) as a parameter, with waveguide dimensions $a / \lambda=b / \lambda=0.5714$, and slab relative permittivity $\varepsilon=3.0625$.

of the finite array) from the total power injected into the finite array. In this way, the power balance theorem is satisfied.

As found in [14], the coupling strength decays rapidly with increasingly displaced elements. For an element spacing even as small as $0.2 \lambda$, the coupling to adjacent neighbors already weakens by $15 \mathrm{~dB}$, and drops to as low as $-40 \mathrm{~dB}$ for the next further away element, i.e., just the second element. Therefore, any one element in a finite array only "feels" its nearest neighbors and does not "sense" the presence of elements that are more than just a few elements away from it. This fact validates the present approach entailing the summation over just a finite but large number of embedded elements that are present in the large arrays considered here $(21 \times 21)$, since most of the interior elements will indeed "sense" its true finite-array environment. As for the edge excited ones, an additional surrounding track of "dummy" (unexcited) elements along the periphery of the excited portion of the array is included in the analysis and computations. By taking the coupled powers (especially from the excited edge elements) lost into these dummy boundary elements into account, the behavior of the excited elements near the edge of the subarray can be more accurately modeled. In view of the rapidly decayed coupling strength as mentioned above, the peripheral "dummy" track only needs to be a few elements thick (4 as treated here).

\section{VAlidation of the TREATMENT APPROACH}

The foregoing theoretical formulation for the RPA-covered waveguide array can also be applied to the treatment of arrays covered by plain (unprinted) dielectric sheets, by simply removing the electric current source contributions of the metal patch, along with appropriate adjustments to the matrix equation. Since this entails only a simplification of the above concepts, the details for this will not be provided here. Instead, numerical results of plain-sheathed waveguide arrays obtained using the present numerical technique will hereby be presented and validated with existing literature.

Generated using our computer code based on the present treatment approach, the right-hand side plots of Fig. 3 show 


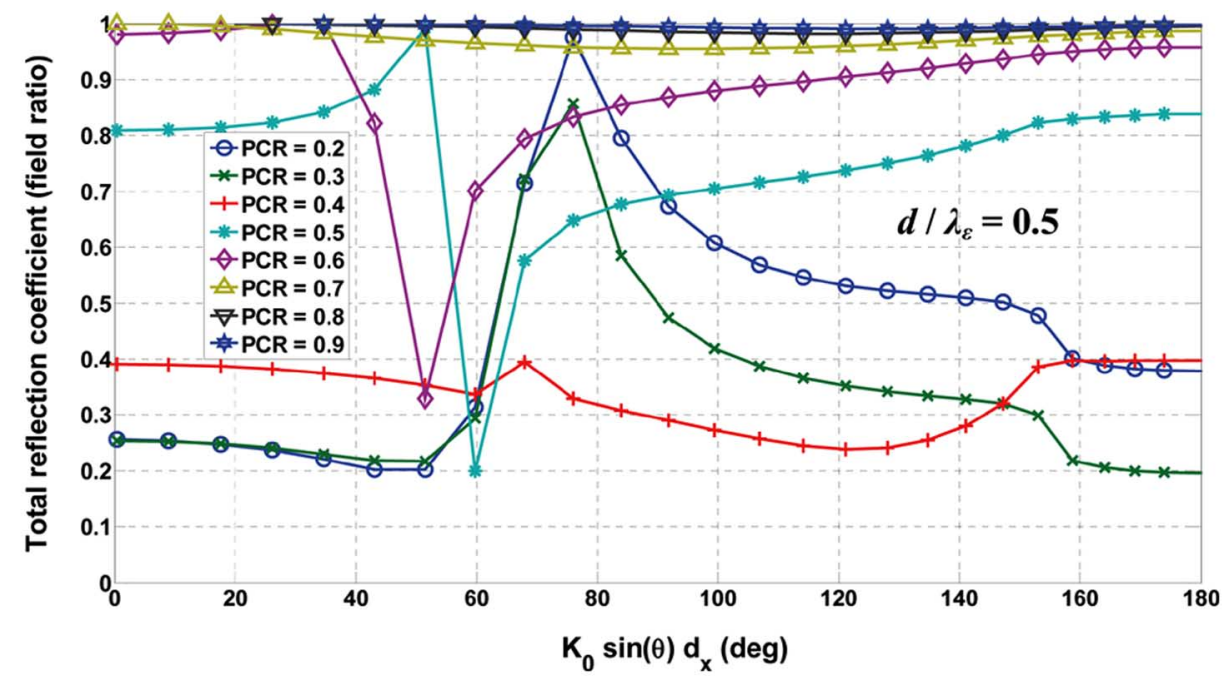

(a)
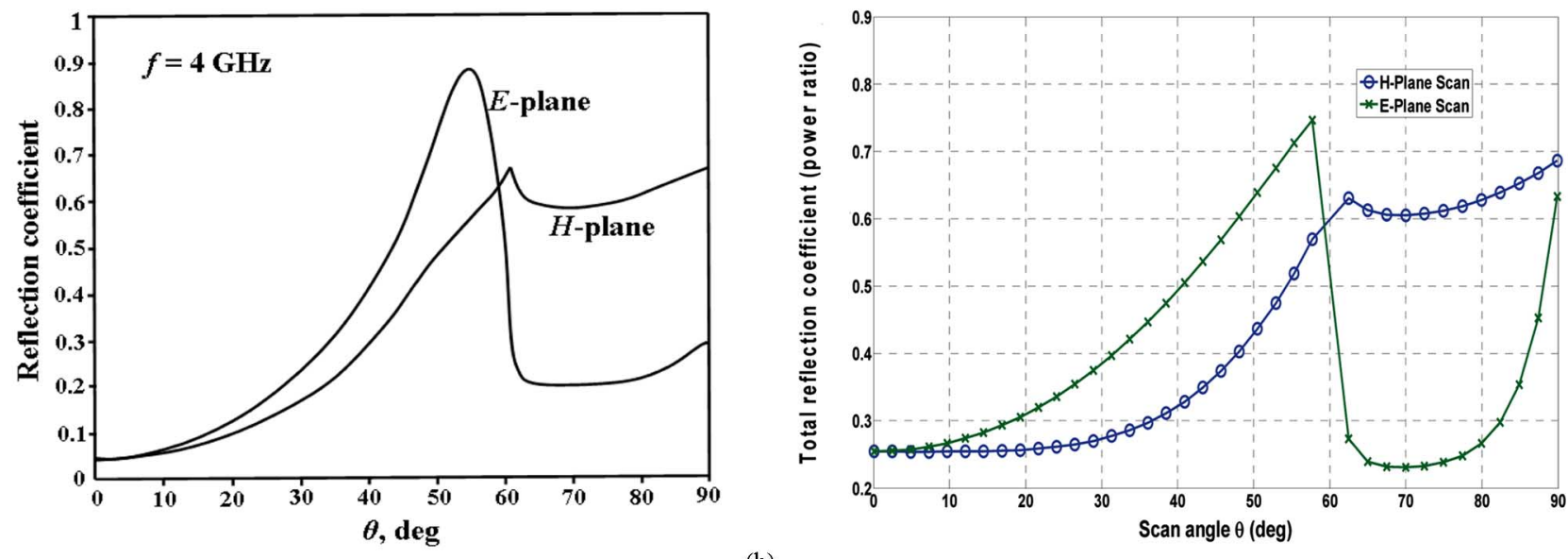

(b)

Fig. 4 (a) DRPA reflection coefficient versus electrical phasing angle $k_{0} d_{x} \sin \theta$ (where $d_{x}$ is the period along $x$ ) of RPA-covered empty rectangular waveguide array for various patch cell ratios $\left(\mathrm{PCR}=l_{x} / d_{x}=l_{y} / d_{y}\right.$ ), for $H$-plane scan and with slab thickness $d=0.5 \lambda_{\varepsilon}$ (where $\lambda_{\varepsilon}=\lambda_{0} / \sqrt{ } \varepsilon$ ), with waveguide dimensions $a / \lambda=b / \lambda=0.5714$, and slab relative permittivity $\varepsilon=3.0625$. The approach towards the corresponding slab thickness case of Fig. 3 as the patch size reduces is evident. (b) Validation results of [16, Fig. 7.4] shown by the left-hand side plot (probe-fed array), reconstructed as the right-hand side plot obtained by our code (waveguide-fed). Graphs of DRPA reflection coefficient versus scan angle $\theta$ of RPA-covered HRW array in $E$ - and $H$-planes at $4 \mathrm{GHz}$, for $d_{x}=d_{y}=$ $4 \mathrm{~cm}, l_{x}=l_{y}=2 \mathrm{~cm}, d=0.7 \mathrm{~cm}$, and $\varepsilon_{r}=2.33$. The dimensions along $x$ and $y$ of the waveguide aperture are 0.85 times $d_{x}$ and $d_{y}$, respectively.

the graphs of the DRPA reflection coefficient against the electrical phasing angle $\left(k_{x 0} d_{x}=k_{0} d_{x} \sin \theta \cos \varphi\right.$ with $\left.\varphi=0\right)$ for $\mathrm{H}$-plane scan of a sheathed rectangular waveguide array, for various slab thicknesses normalized to the dielectric wavelength: $\lambda_{\varepsilon}=\lambda_{0} / \sqrt{ } \varepsilon$, where $\varepsilon=3.0625$ is the relative permittivity of the plain sheet, and with $a / \lambda_{0}=b / \lambda_{0}=0.5714$ being the waveguide dimensions along the two principal planes of the array aperture, normalized to the free space wavelength $\lambda_{0}$. This configuration is according to [21, Figs. 6.12 and 6.13], which are replicated here as the left-hand side plots of the same Fig. 3. The upper and lower plots here correspond to [21, Figs. 6.12 and 6.13], respectively. As can be clearly seen, the agreement of our results with those of that literature source is excellent. It is noted that the waveguide elements here are empty, i.e., not loaded with any dielectric. Hence, our robust code can treat any kinds of dielectric loadings, be it partially sidewall-loaded (as focused here), fully dielectric filled, or completely empty.
There are no known prior works published on RPA-covered waveguide arrays. Nonetheless, an alternative way of validating the technique for treating such structures is offered here-by demonstrating how the results of RPA-covers approach those of unprinted plain sheets (which have been verified above) as the size of the patches is progressively reduced. This is presented in Fig. 4(a), which shows the same kind of graphs as Fig. 3, but this time for RPA-covered arrays of various patch sizes. With the exception of the patches, all other parameters of the array and dielectric slab remain as before in Fig. 3. Evidently, as the patch gets smaller, the results approach those of the unprinted slab. This approach is deduced to be fairly abrupt, in the sense that, only for patch cell ratios $\left(\mathrm{PCR}=l_{x} / d_{x}=l_{y} / d_{y}\right.$ ) below 0.4 do the results resemble those of the unprinted sheet. Observing further, when the patch is large compared to the unit cell (PCR close to unity), the phased array reflection coefficient is high over all scan angles, which is as expected. 
Another improvised validation of the RPA-covered array treatment is now provided. A probe-fed patch array has been studied in [16, ch. 7]. Although the excitation probe there is different from our feed waveguide here, we will still conduct a comparison study. The right-hand side plot of Fig. 4(b) depicts the graph of phased array reflection coefficient versus scan angle for $E$ and $H$ planes at $4 \mathrm{GHz}$, generated using our code for treating RPA-covered waveguide arrays, for the same patch array configuration as of [16, Fig. 7.4], conveyed here as the left-hand side plot. Specifically, according to our notations here (see Fig. 1), $d_{x}=d_{y}=4 \mathrm{~cm}, l_{x}=l_{y}=2 \mathrm{~cm}, d=0.7 \mathrm{~cm}$, and $\varepsilon_{r}=2.33$. The dimensions along $x$ and $y$ of our HRW aperture are 0.85 times $d_{x}$ and $d_{y}$, respectively, the sidewall loading thickness is 0.1 times the aperture width, and its permittivity is such that the waveguides are hard at the investigated $4 \mathrm{GHz}$. Clearly, there are very strong similarities between our results and those of [16], in terms of both trends and levels. In fact, the only difference here is that the reflection level of our $E$-plane scan rises sharply as the scan angle exceeds $80^{\circ}$ and approaches grazing, whereas it remains fairly low up until $90^{\circ}$ in $[16$, Fig. 7.4]. Nevertheless, considering the difference in the way the patch arrays are fed, such a minor dissimilarity is deemed trivial. As a by-product found here, it can be deduced that the characteristics of patch arrays are predominantly determined by their own properties, and less influenced by the way they are fed.

\section{NUMERICAL RESULTS AND DISCUSSION}

All numerical results presented herein for the RPA-covered as well as the plain-sheathed HRW array were generated by the moment method code developed according to the analyses and concepts of Sections II and III. It is reminded that the plainsheeted arrays are also solved using those same formulations and theories, but in a simplified form (removal of the PEC patch contributions). The computed data for HRW arrays without any form of cover were also obtained by moment method codes constructed in [14].

\section{A. Universal Conditions: HRW Array and FPA Scenario}

The following description of the HRW array parameters and the conditions of the FPA scenario pertains to all forthcoming computed results. The geometry and parameters of the investigated HRW arrays are first defined. A square unit cell is considered, with periods $d_{x}$ and $d_{y}$ along $x$ and $y$ being $d_{x}=d_{y}=$ $6.9 \mathrm{~mm}$. The likewise square HRW aperture has dimensions along either $x$ or $y$ being 0.98 times of $d_{x}$ or $d_{y}$, respectively. Hence, the elemental HRW aperture and unit cell sizes are fixed at all frequencies. As for the dielectric sidewall-loaded HRWs, the ratio of the side-slab thickness (any one of the two slabs) to the total waveguide width is maintained at $1: 40$ throughout. As the frequency varies, the permittivity of the dielectric sidewall loading changes in such a way that the HRW elements are always operated at the transverse electromagnetic (TEM) hard condition, i.e., every frequency computed is the TEM frequency [10]. It has been shown in [10] that the HRW array is well matched when the elements are operated at the TEM frequency. Therefore, the HRW array studied here is optimized for every investigated frequency. The reason for this selection is to make the performance of the matching layer independent of the array element. In this manner, the computed performance of the matching layer is dependent only on its own parameters, and the presented results reflect its true performance. Throughout the numerical investigations, 30 waveguide modal basis functions are used for expanding the waveguide aperture magnetic current, and 11 RWG basis functions expand the electric patch current. A total of 169 Floquet harmonics are entailed in the discrete spectrum. These parameters were determined by a convergence study, thus assuring that accurate and converged results are obtained.

Coming now to describe the FPA scenario, consider a paraboloidal reflector antenna illuminated by an incident on-axis plane wave. For shallow dishes, the resultant focal plane fields approaches a form of the Airy pattern, which comprises a central main lobe and surrounding concentric sidelobe rings. When a finite number of elements of the RPA-covered HRW array are excited according to the levels of this Airy pattern at the respective center locations of the elements in the focal field distribution, in a discretized and truncated fashion [14], the array operates as an FPA feed for that parabolic reflector for radiating an axial beam. In the present study, a finite number of $21 \times 21$ elements are engaged in sampling the Airy pattern. A paraboloid with $60^{\circ}$ half subtended angle and a diameter of $5 \mathrm{~m}$ is considered here. As such, as the frequency varies, the extent of the Airy pattern which the finite and constant-sized FPA samples also varies.

\section{B. Comparison of Uncovered HRW Arrays With Plain Sheathed and RPA-Covered Arrays}

The comparison of the radiation efficiencies between uncovered HRW arrays and those covered by plain dielectric sheets or RPA layers is now performed. For the upcoming initial studies (more cases will be considered later), three dielectric slab thicknesses $d$ of 1.13, 2.25, and $3.375 \mathrm{~mm}$ are studied. For the sheath type, three relative permittivities of $2.2,3$, and 4 are investigated, whereas for the RPA layer, a fixed relative permittivity of 2.2 is maintained, but the size of its square metal patch varies over $l_{x}=l_{y}=2.07,4.14$, and $6.1 \mathrm{~mm}$, where $l_{x}$ and $l_{y}$ are the lengths along $x$ and $y$.

Before proceeding further, a proper definition of the term "bandwidth" that will be used in the remaining parts of the paper is in order. The "bandwidth" is meant here as the "matching bandwidth," which is defined as the frequency range over which the transmission of the array covered by a matching layer (plain sheet or patch array) surpasses that of the corresponding uncovered array.

1) Uncovered Versus Plain Sheets: The coupling efficiencies of plain sheet covered FPAs excited according to the Airy pattern are compared with those of an uncovered one in Fig. 5(a) over a frequency range of 2-26 GHz. The matching performances of the sheath tuners are found to be good at low frequencies when their thicknesses are large, whereas thin sheaths are superior at high frequencies. This suggests that for any one particular array configuration, there is an optimal slab thickness in terms of dielectric wavelength $\lambda_{\varepsilon}=\lambda_{0} / \sqrt{ } \varepsilon_{r}$ which gives the best matching. Hence, while the thick sheaths are closer to the optimal electrical slab thickness at low frequencies, it is 

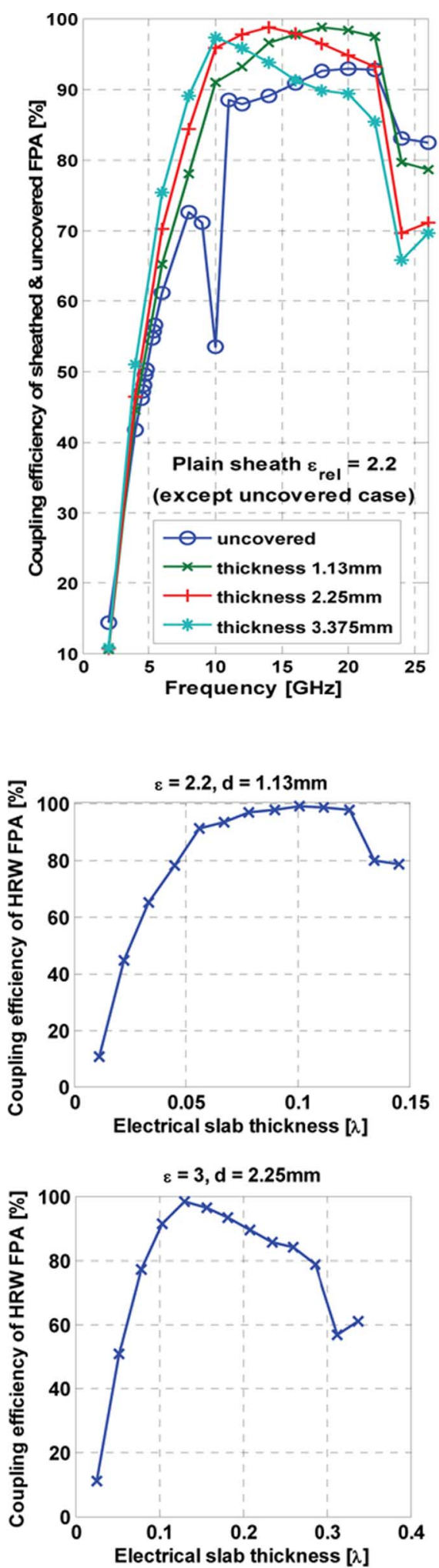

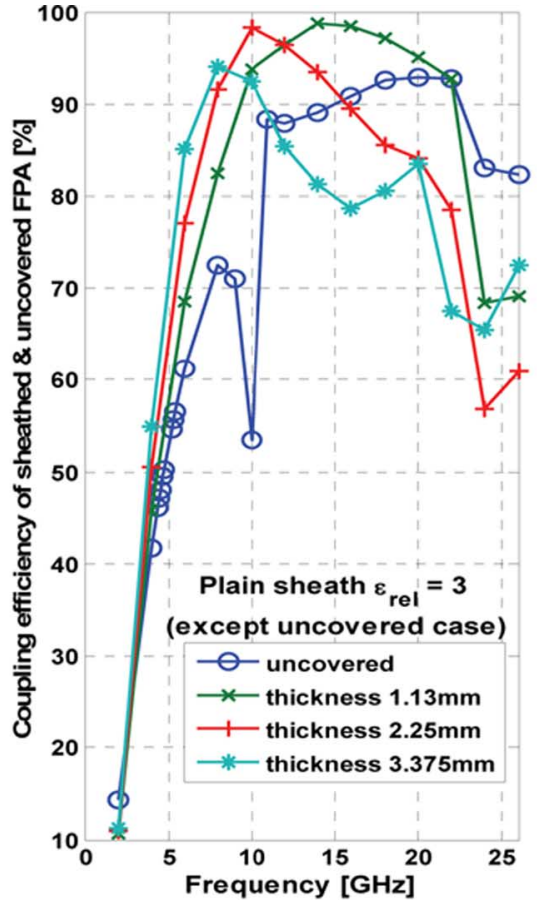

(a)
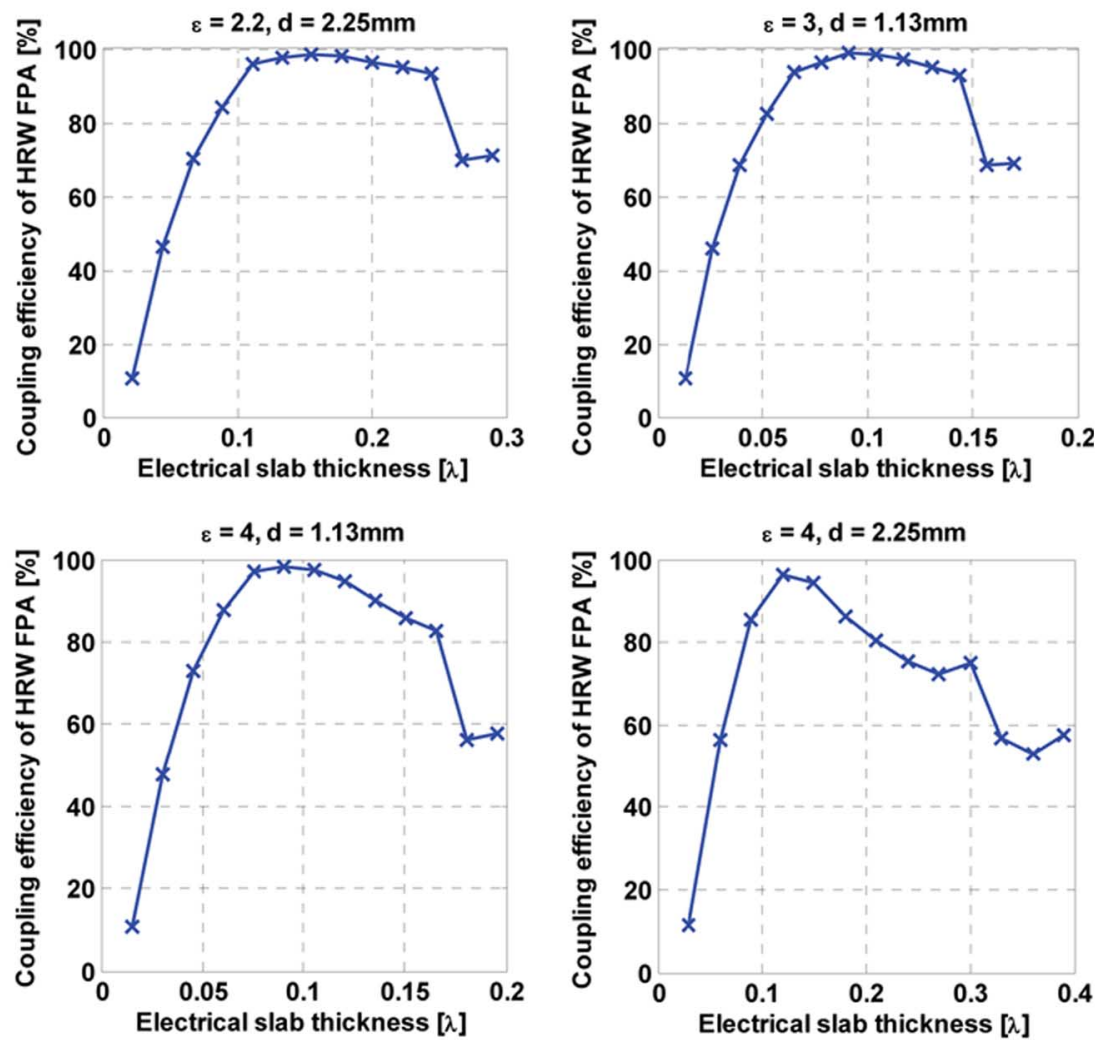

(b)
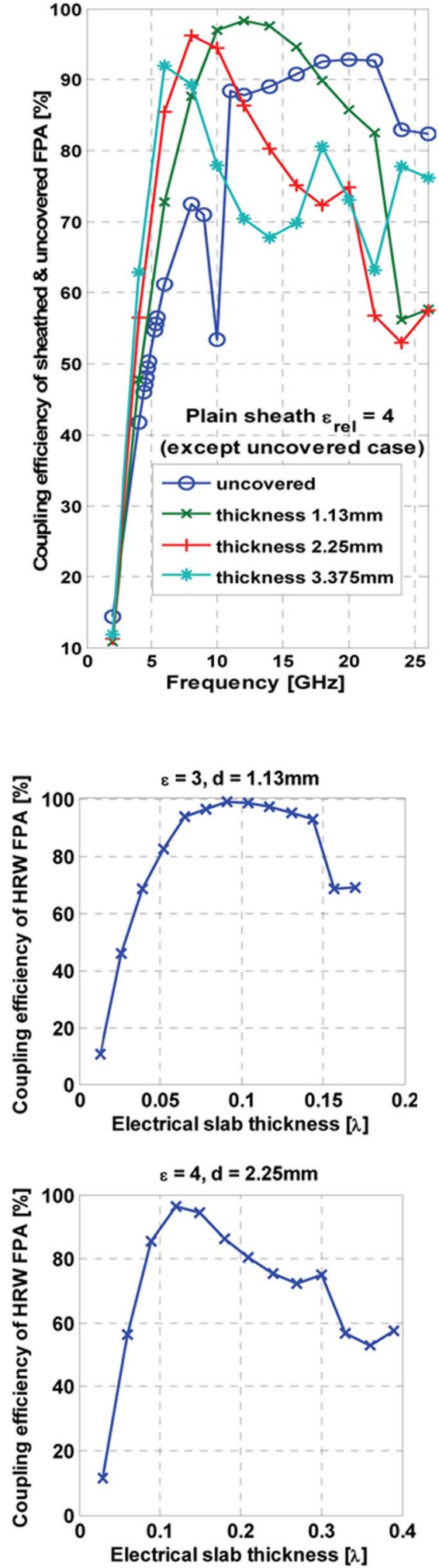

Fig. 5 (a) Variation of radiation performance with frequency for sheath-covered HRW array operating as FPA (for 5-m $60^{\circ}$ paraboloid). (b) Variation of radiation performance with electrical slab thickness for sheath-covered HRW array operating under FPA scenario (for 5-m $60^{\circ}$ paraboloid). A universal optimum at around $0.1 \lambda$ electrical slab thickness is apparent.

the thin ones that are closer to it at high frequencies. The existence of an optimal electrical slab thickness is demonstrated in Fig. 5(b), which presents the subplots of Fig. 5(a) as func- tions of electrical slab thickness. For all cases, a universal optimum of approximately $0.1 \lambda$ slab thickness is apparent. It is also noticed that although thick slabs are more effective at low 

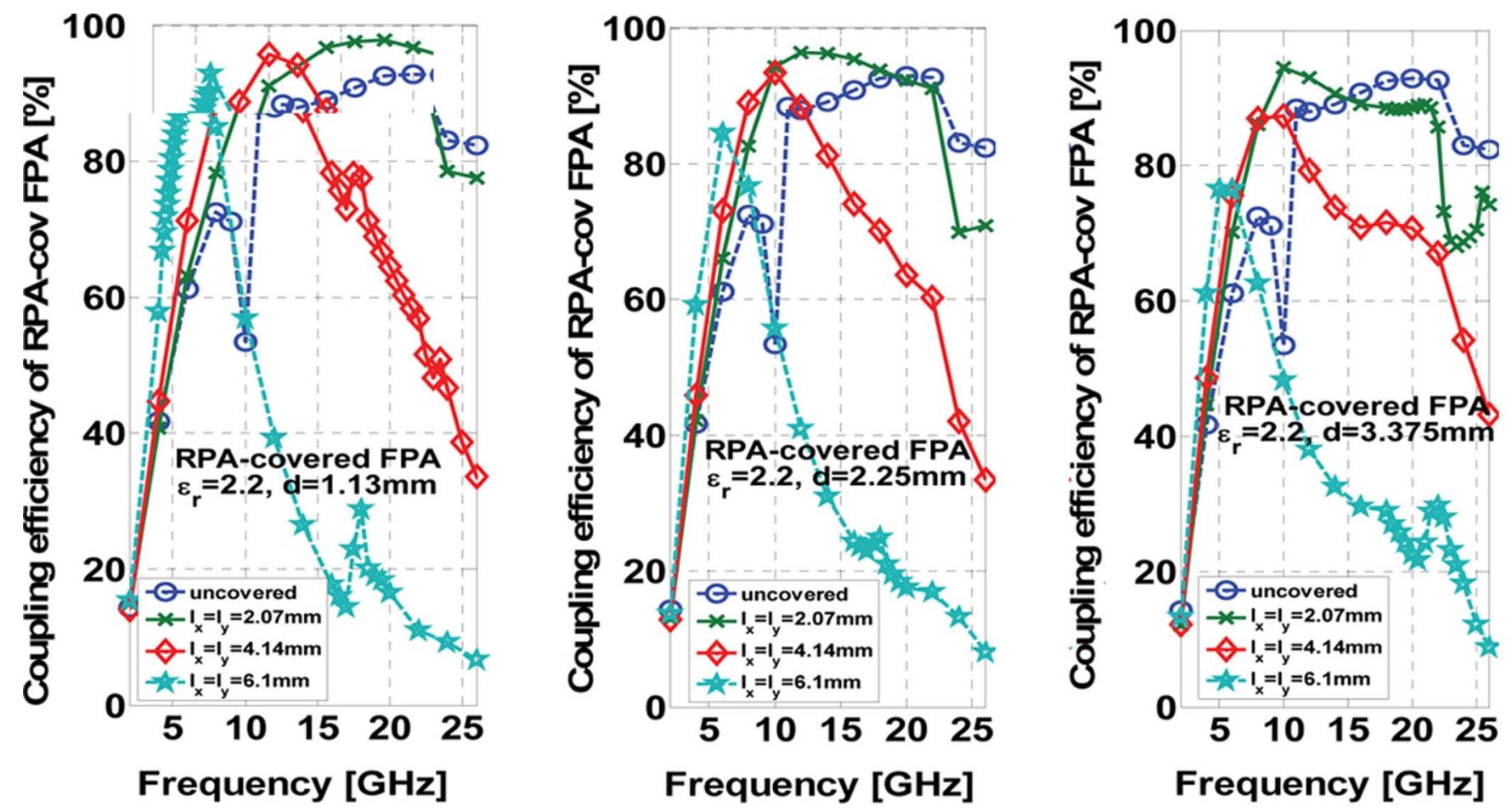

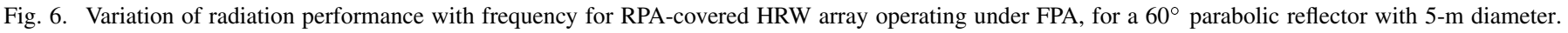
A universal slab $\varepsilon_{r}=2.2$ is used.

frequencies, their rapidly degraded performances at high frequencies make their matching bandwidths narrower than those of thin sheets. The sharp drops in efficiency levels at occasional frequencies in some cases are due to blindness effects common in phased arrays. The active reflection coefficients of such conditions for certain scan angles become large, thus leading also to very high mutual coupling coefficients for the embedded element scenario. This is subsequently translated to low coupling efficiencies of the finitely excited FPA scenario.

Fig. 5(a) may be manifested in another way by swapping the two parameters, namely, the sheet thickness and permittivity. Due to space constraint, such reconveyed graphs cannot be presented here. But just verbally, it will be seen that the same effects mentioned above caused by thick and thin slabs can be translated to high and low slab permittivities, respectively. As before, the notion of an optimal electrical slab thickness can be put forward. This time, however, since the slab thickness of each subplot is now constant, an optimal dielectric wavelength $\lambda_{\varepsilon}$ is perceived instead. At low frequencies, a correspondingly large $\varepsilon_{r}$ is needed to keep up with the large $\lambda_{0}$ to maintain a certain optimal $\lambda_{\varepsilon}$, and vice versa.

Therefore, from these discussions, we have found that by tailoring the slab thickness or permittivity of the plain sheath layers, either low-frequency matching or broadband matching can be achieved, although there is a tradeoff between them.

2) Uncovered Versus RPA Covered: The matching capabilities of RPA layers are illustrated in Fig. 6 for the FPA scenario. It is seen that large metal patches (relative to the fixed unit cell) achieve low frequency but narrowband matching, whereas smaller ones are broader band (particularly effective at high frequencies) but not as capable in their low-frequency matching.
Hence, increasing the metal patch size of the RPA layer has the same effects as increasing the permittivity of a plain dielectric sheath. The peak achievable radiation levels are also higher for smaller patches. As was for the preceding case, the plots of Fig. 6 may be represented in another format by swapping the parameters, namely, the patch size and slab thickness. Like before, however, such graphs are not provided here due to space limitation (although they are just based on the same numerical data of Fig. 6 anyway). Nonetheless, it is just described with words here that from such plots, it will be observed that the performance at low frequencies (below $5 \mathrm{GHz}$ ) appears to be unchanged regardless of the slab thickness. Thus, unlike the plots of Fig. 5(a), increasing the slab thickness now does not result in improved (but neither degraded) low-frequency matching, but still leads to narrower bandwidth, whereas thin slabs are better at higher frequencies and are broader band. Moreover, the peak attainable radiation performance seems to be better when the slab is thin.

3) Plain Sheets Versus RPA Covers: As mentioned earlier, depending on the required functionality, i.e., whether low-frequency or broadband array matching is desired, the parameters of the two forms of matching layers may be tailored to meet the specifications. We will now consider these two possible performance requirements separately, and then select from our presently computed set of cases the best performers of the two classes of matching layers for comparison.

Let us first consider low-frequency matching as the requirement. Based on the parameter space of the foregoing two Sections V-B1 and V-B2, we know that a sheath with the thickest slab and highest permittivity would be most effective, whereas an RPA layer with the largest patch size and thinnest 
slab will be optimal. Note that the latter factor is chosen to optimize the maximum attainable performance, since varying the slab thickness of the RPA layer has negligible effects on the low-frequency matching, as mentioned earlier. Therefore, it will be the sheath with relative permittivity of 4 and thickness of $3.375 \mathrm{~mm}$, whereas the RPA layer in contention has patch size of $l_{x}=l_{y}=6.1 \mathrm{~mm}$, slab thickness $1.13 \mathrm{~mm}$, and the standard $\varepsilon_{r}=2.2$. Fig. 7(a) shows the radiation performance of the arrays covered by these two forms of matching structures over a frequency range, for the FPA scenario. The performance of the uncovered array is, of course, also included. Evidently, the performances at low frequencies for both operation scenarios have been significantly improved by both tuners, and they are seen to be comparable. However, the sheath tuner is superior at high frequencies and thus in terms of bandwidth, although now we are only looking at low-frequency matching. Nevertheless, beyond $11 \mathrm{GHz}$, neither structure serves to improve the uncovered array.

Where broadband matching is concerned, the preceding Section V-B2 dictates that a sheath with the thinnest slab $(1.13 \mathrm{~mm})$ and lowest permittivity (2.2) is required, while an RPA layer with the smallest patch size $\left(l_{x}=l_{y}=2.07 \mathrm{~mm}\right)$ and thinnest slab $(1.13 \mathrm{~mm})$ is needed. The wideband matching of these layers is conveyed in Fig. 7(b). Indeed, with these matching covers, the radiation capability of the uncovered array is improved over a vast range of frequencies $(2-22 \mathrm{GHz})$, although the benefit is less pronounced at low frequencies. This again underlines the tradeoff between low-frequency and wideband matching.

Through this investigation, the usefulness of these two forms of impedance match tuners has been ascertained. This work thus marks an inaugural effort towards the impedance matching of FPAs, especially for the lower frequency bands, in which the problems associated with the increased severity of mutual coupling losses have been of primary concern.

a) Bandwidth Study of Nonideal Waveguide Array Elements: As stated earlier, the waveguide elements are operated at the TEM condition for every investigated frequency over a wide band, the purpose of which is to demonstrate solely the effectiveness of the matching layers, but with the varying influences of the array elements at different frequencies being minimized. This then entails a disparate value of the permittivity of the dielectric sidewall loading inside the waveguide elements at each frequency. However, in order to gain insights into the true matching bandwidth of realistic elements having fixed dielectric loadings, we hereby conduct bandwidth studies of actual invariable waveguide elements about two showcased frequency bands: a low one of $6 \mathrm{GHz}$, and another at $12 \mathrm{GHz}$, using the same matching layers prescribed by Fig. 7(a) and (b), respectively. Hence, the only difference now is that the waveguide elements are dielectrically sidewall loaded in such a way that they are hard (TEM) only at the center frequency of the investigated band, i.e., 6 and $12 \mathrm{GHz}$.

The results for these bandwidth studies are presented in Fig. 8. The thickness $t$ of each sidewall dielectric loading in the waveguide element is now fixed at $1 \mathrm{~mm}$, as opposed to $(6.9 \times 0.98) / 40 \mathrm{~mm}$ as considered earlier, and its relative permittivity is $\varepsilon_{r, w g}=1+\left[\lambda_{\mathrm{TEM}} /(4 \mathrm{t})\right]^{2}$, where $\lambda_{\mathrm{TEM}}$ is the

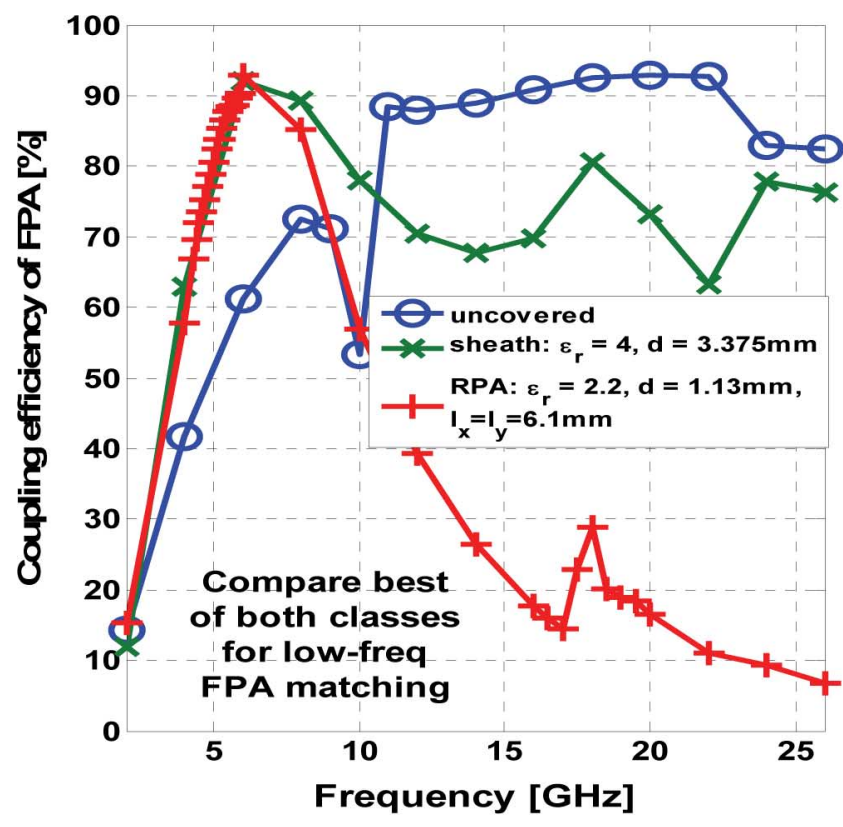

(a)

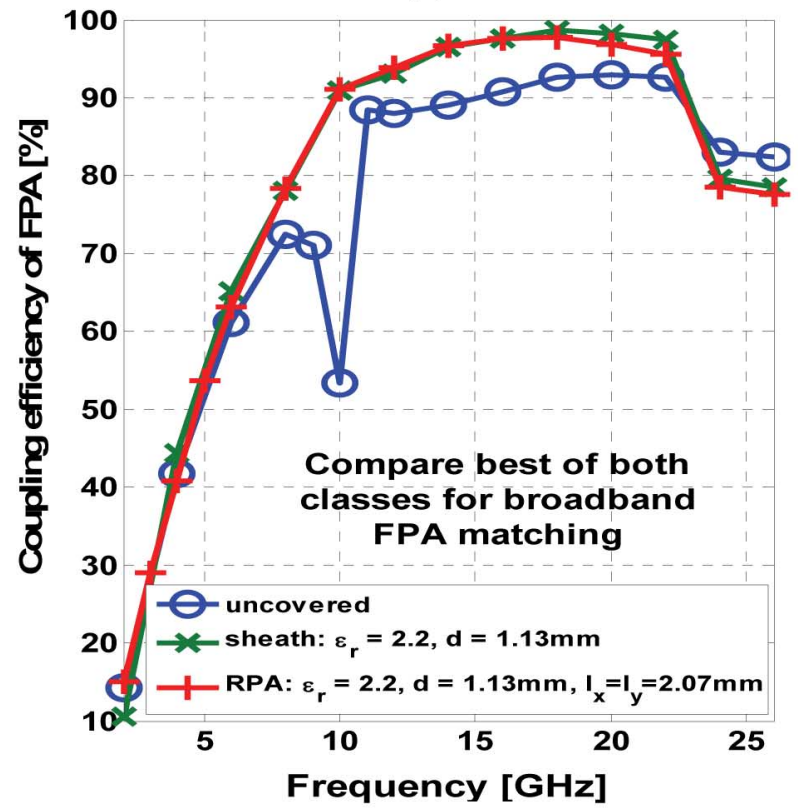

(b)

Fig. 7. Comparison of (a) low-frequency matching, and (b) broadband matching capabilities between the best performers of both classes of matching structures, for FPA scenario.

free space wavelength at the TEM frequency, being either 6 or $12 \mathrm{GHz}$ as studied here. The subscript $w g$ is to distinguish this sidewall loading of the waveguide from the $\varepsilon_{r}$ of the overlaying dielectric covers. Fig. 8(a) shows the performances of the same three matching structures as Fig. 7(a), over the range from 5.9 to $6.2 \mathrm{GHz}$. As seen, the two types of matching layers can indeed enhance the radiation of their uncovered counterpart over this entire band. The coupling efficiency of the uncovered FPA is increased by at least $25 \%$ throughout the investigated band, which is a significant improvement. The matching effectiveness of both covers is also almost equal, which is as 


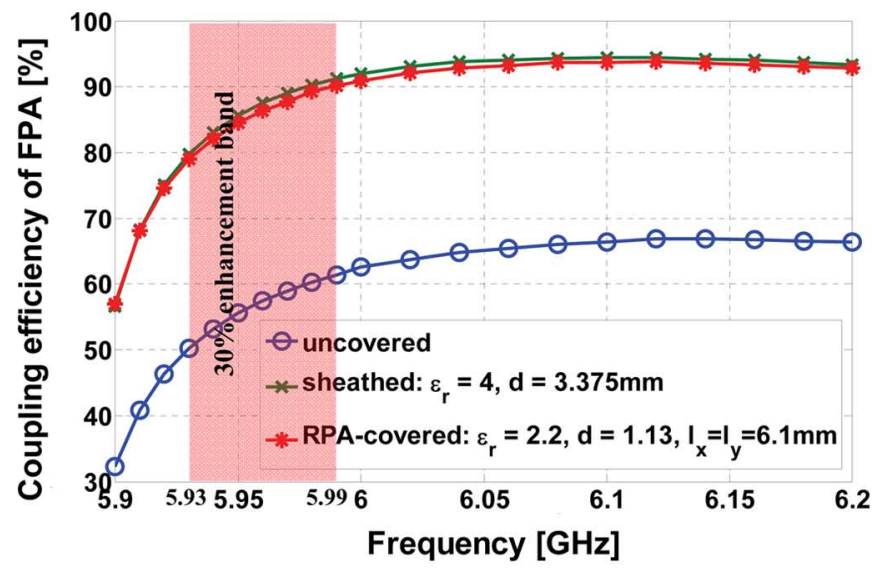

(a)

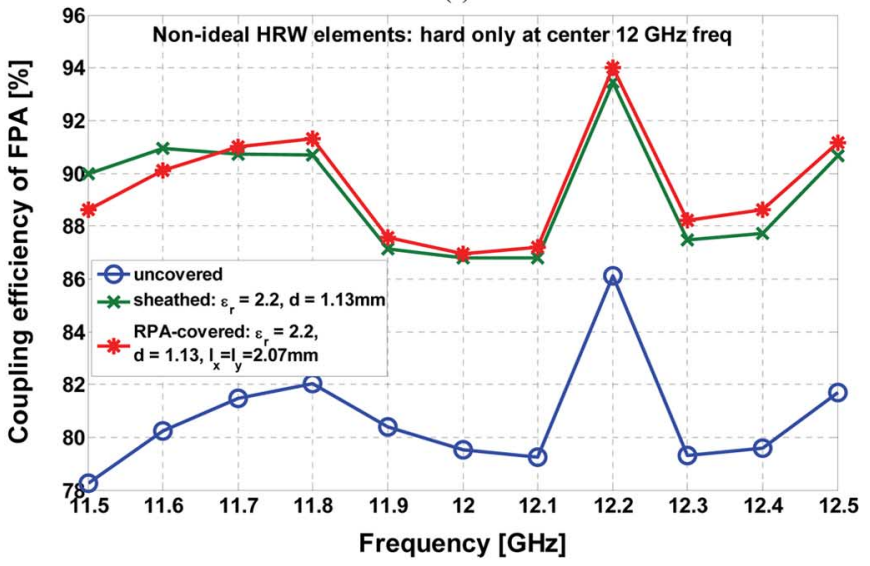

(b)

Fig. 8. Bandwidth studies about (a) $6 \mathrm{GHz}$, and (b) $12 \mathrm{GHz}$, for nonideal (realizable) HRW FPA, with matching covers as prescribed by Fig. 7(a) and (b), respectively, for low-frequency and broadband matching. The sidewall dielectric thickness of the waveguide element is maintained at $1 \mathrm{~mm}$, and it is hard only at the respective center frequencies.

expected due to their similar performance levels at $6 \mathrm{GHz}$ in Fig. 7(a). However, the weight and profile advantages of the RPA-cover are apparent. The corresponding study at $12 \mathrm{GHz}$ is given in Fig. 8(b), for the range from 11.5 to $12.5 \mathrm{GHz}$. Likewise, the configurations of the two matching layers here are the same as those of Fig. 7(b). Again, there is considerable improvement of the uncovered FPA coupling efficiency-by at least $7 \%$ throughout the band, and both covers perform at similar levels. Therefore, these results show that there is an approximate $1 \%$ matching bandwidth with $30 \%$ improvement in coupling efficiency achieved by both the plain sheath and RPA cover about a $5.96-\mathrm{GHz}$ center frequency, and at least an $8.3 \%$ enhancement bandwidth (with $7 \%$ increase) at $12 \mathrm{GHz}$.

\section{Superior Matching With Lower Profile, Wider Band RPA Covers}

Two aspects have been learned from the preceding subsection: 1) there is an optimal electrical thickness of the dielectric sheet tuner, and 2) the size of the metal patch of an RPA cover has a correspondence with the permittivity of an unprinted sheath. The former leads to the following. At low frequencies, an unprinted board of either, 1) low permittivity but large thickness, or 2) small thickness but high permittivity, is needed. However, each option has its drawback; the former suffers from high-profile and weight problems, whereas the latter encounters bandwidth and loss issues. Capitalizing on the second finding stated above, these challenges posed towards low-frequency matching may be met by using a thin slab of low permittivity printed with an array of large patches (increases the effective permittivity). This is in view of the fact that the metal patch array incurs virtually zero cost in terms of thickness and weight.

Therefore, this section seeks to ascertain the equivalence in the radiation performance of an array covered by a thick unprinted board of low permittivity and an RPA cover with a thin slab of that same low permittivity but printed with large patches. In addition, for a particular prescribed performance level (coupling efficiency) at a certain low frequency, the matching bandwidth between a thin plain sheet of high permittivity and an RPA cover comprising a thin slab of low permittivity and large patches (both achieving that performance level at that low frequency) will be compared.

For a fixed slab relative permittivity of 2.2 , being a low value, Fig. 9(a) shows the variation of the coupling efficiency of a plain-sheathed HRW FPA with the sheet thickness for several low frequencies. As can be seen, at low frequencies, the radiation level rises steadily with the board thickness, in accordance with the theory just described. An optimal thickness exists for each frequency, as explained earlier, but does not show up in the considered range of thickness for low frequencies. At higher frequencies, however, the optima are lower, thus appearing as shown. The maximum achievable efficiency is higher for higher frequencies, due to reduced mutual coupling losses between elements.

The corresponding variation of the radiation performance with the patch size of the RPA cover relative to the unit cell is given in Fig. 9(b), again for various low frequencies. Here, the slab thickness is low, at $1.13 \mathrm{~mm}$, and the same $\varepsilon_{r}=2.2$ is used. As expected, the efficiency rises as the patch size increases, due to the increased effective permittivity. Hence, we have demonstrated that using an RPA cover with a thin slab printed by large patches provides similar tuning effects at low frequencies as a thick dielectric sheet, both structures sharing the same slab material. Observing further, it is found that, for frequencies below $5 \mathrm{GHz}$, the maximum efficiency achievable by both matching structures is approximately the same (e.g., at $2 \mathrm{GHz}$, both can attain around 15\%, at $3 \mathrm{GHz}: 40 \%$, and at $4 \mathrm{GHz}$ : 60\%). However, while the maximum level drops beyond a certain plain sheet thickness (due to the presence of the optimal thickness as explained above) for higher frequencies, the performance of the RPA cover does not suffer from such falloff conditions, i.e., the rise in efficiency is maintained all the way up to large patches at higher frequencies. In fact, the rate of climb is seen to be increasing even. As a result, the maximum achievable efficiency of the RPA-covered array at $5 \mathrm{GHz}$, being around $80 \%$, is superior to the $75 \%$ attainable by its plain sheet alternative. Likewise, an approximate $95 \%$ maximum level of the RPA-cover at $6 \mathrm{GHz}$ far exceeds the $80 \%$ of the corresponding sheet. Therefore, in addition to the lower profile and lighter weight advantages at low frequencies, the matching performance of RPA-covered arrays surpasses those 


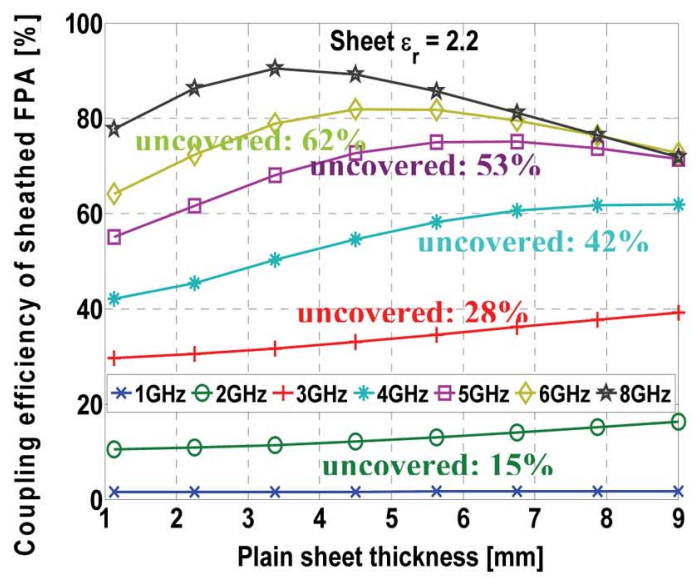

(a)

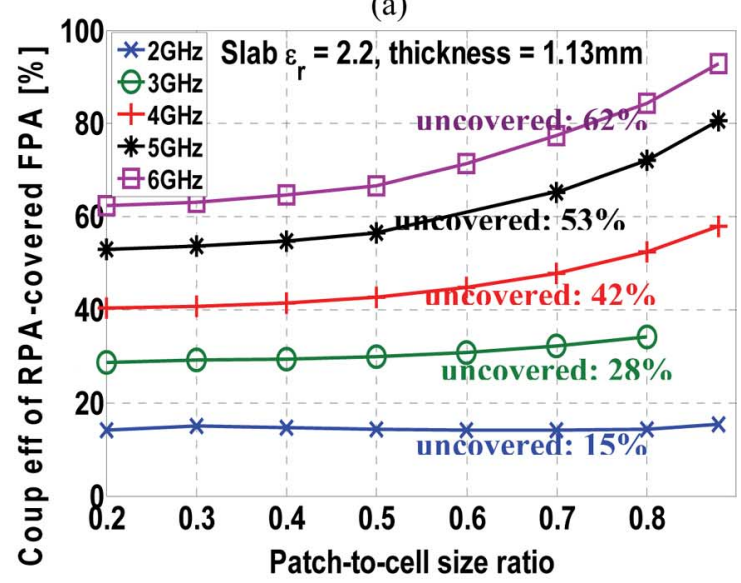

(b)

Fig. 9. Variation of (a) sheathed FPA coupling efficiency with slab thickness, and (b) RPA-covered FPA coupling efficiency with PCR, both for various frequencies, for a fixed slab relative permittivity 2.2. The fixed slab thickness of the RPA cover is a low value of $1.13 \mathrm{~mm}$. The efficiencies of the uncovered FPA at the investigated frequencies are indicated along each associated curve.

of the plain sheets at intermediately low frequencies, e.g., between 5 and $6 \mathrm{GHz}$.

As a final observation of Fig. 9(a) and (b), the efficiencies of RPA-covered FPAs using the smallest patch-to-cell-size ratio [patch cell ratio $=0.2$ in Fig. 9(b)] at various frequencies are approximately the same as those of plain sheathed FPAs using the thinnest sheet $[d=1.13 \mathrm{~mm}$ in Fig. 9(a)] of the same material as the RPA cover, and being also the board thickness of the latter. This coheres with the phenomenon conveyed by Fig. 4(a) and thus further supports the validity of the computed results.

Fig. 10(a) shows the graph of the plain-sheathed FPA coupling efficiency versus the sheet thickness for various slab permittivities, at a single 5-GHz frequency. As seen, there is a leftward movement of the peaks with increasing value of the parameter $\left(\varepsilon_{r}\right)$. This is directly correspondent to the existence of an optimal electrical sheet thickness, as explained earlier, which can again be calculated to be around $0.1 \lambda_{\varepsilon}$.

To gain some insights into the tolerances and extent in which the RPA cover surpasses the plain sheet, Fig. 10(a) is compared with the 5-GHz trace of Fig. 9(b) for the RPA cover. We will give the plain sheet a handicap advantage over the RPA cover by ignoring the profile factor. It can be seen from Fig. 10(a) that the peak attainable efficiency of the plain sheet can exceed the

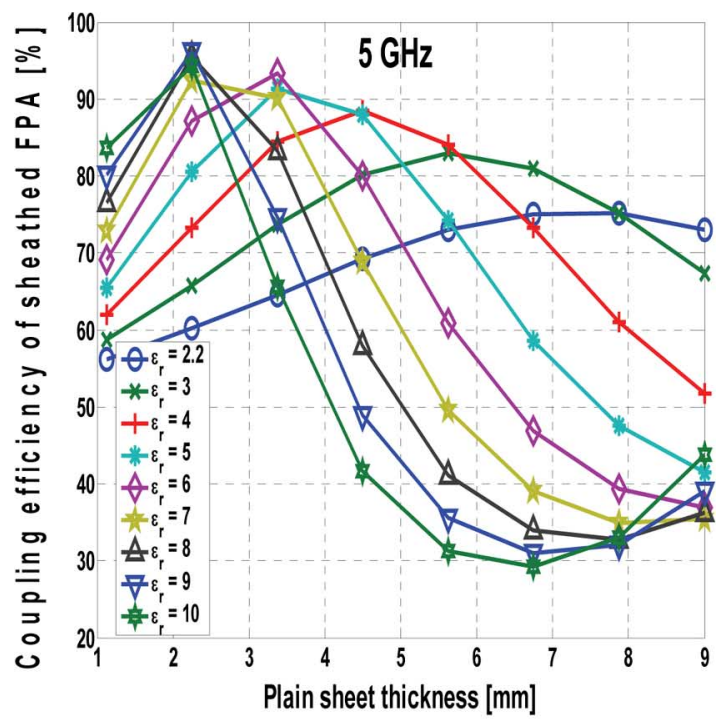

(a)

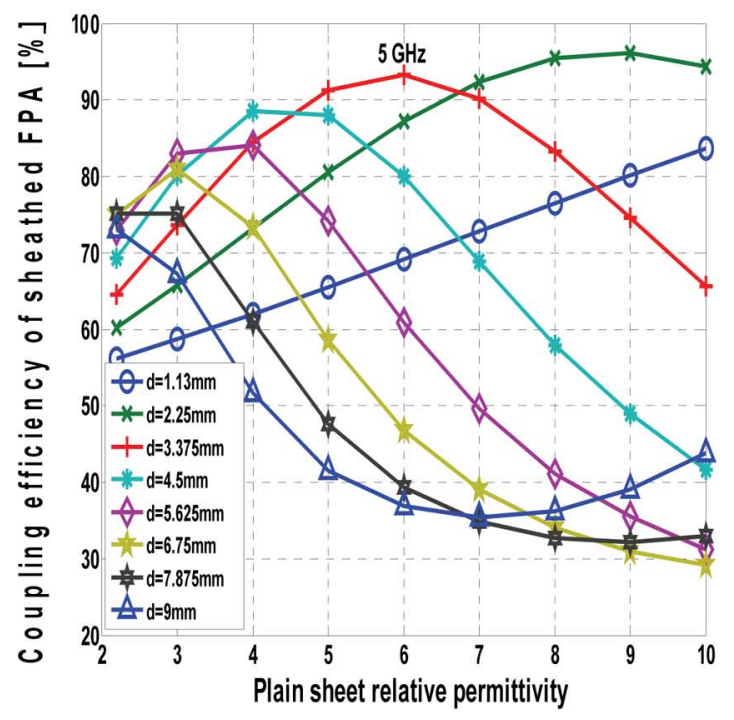

(b)

Fig. 10. Variation of sheathed FPA coupling efficiency with sheet's (a) thickness, and (b) relative permittivity, at $5 \mathrm{GHz}$. Leftward movement of peaks in both graphs with increasing parameter value, in correspondence to optimal electrical sheet thickness.

the $80 \%$ of the RPA cover (with large patch-to-cell-size ratio of 0.884 ) by using boards with higher permittivities and reduced thicknesses - albeit being still higher profile than the RPA cover, which we disregard as said. Hence, the previously stated advantage of the RPA cover over the plain sheet in terms of the peak attainable efficiency can be quite easily neutralized by using higher $\varepsilon_{r}$ values, but provided the profile of the matching structure is not an issue. However, if the profile factor is brought back in for a fair comparison, i.e., remove the handicap, then there is no way the plain sheet can subdue the RPA cover, as can be seen from Fig. 10(a), in which none of the $\varepsilon_{r}$ cases is able to surpass the $80 \%$ at $d=1.13 \mathrm{~mm}$. At best, the sheet with the highest $\varepsilon_{r}=10$ matches the $80 \%$ peak performance of the RPA cover with the same small slab thickness of $1.13 \mathrm{~mm}$. This latter concurs with the earlier stated theory about the need for high permittivity when the sheet is thin. 


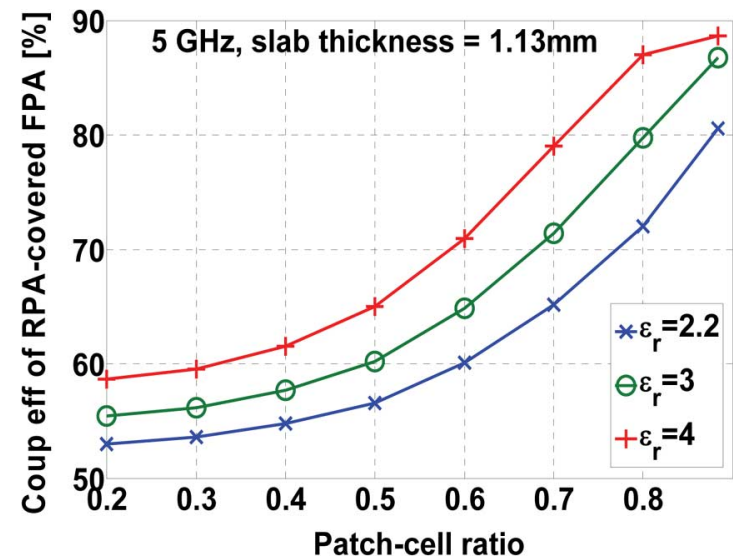

Fig. 11. Variation of RPA-covered FPA coupling efficiency with patch-to-cellsize ratio, for various slab permittivities at a fixed $5 \mathrm{GHz}$ and small slab thickness $d=1.13 \mathrm{~mm}$. The best $90 \%$ performance of plain sheets with high $\varepsilon_{r}$ in Fig. 10 is easily (and rapidly) matched by just mildly increasing the slab $\varepsilon_{r}$ of RPA covers even without needing to increase the thickness.

Fig. 10(b) conveys Fig. 10(a) in another form, this time with the efficiency plotted as a function of the plain sheath permittivity. A likewise leftward shift of the peaks of the curves with increasing sheet thickness is evidence of the optimal electrical slab thickness. Indeed, for thin boards, the radiation performance improves with initially increasing slab permittivity. The rise, however, does not go on indefinitely, as there is a point (peak condition) when the optimal electrical slab thickness is exceeded.

The former comparison gave the plain sheet an unfair advantage over the RPA cover by increasing its permittivity above that of the slab of the RPA cover and neglecting the profile factor. If, however, the slab permittivity of the RPA cover is also allowed to increase, for a fairer comparison, the regaining of its advantage over the plain sheet is very rapid, even without a need for raising its slab thickness. This is demonstrated in Fig. 11, which shows the same graphical format as of Fig. 9(b), but this time only for a single $5 \mathrm{GHz}$ and various $\varepsilon_{r}$ (the low $d=1.13 \mathrm{~mm}$ remains universal). The previous superiority in terms of the peak attainable efficiency (although on unfair grounds) of the plain sheets with $\varepsilon_{r}$ larger than 3 seen in Fig. 10(a) over the best RPA cover at $5 \mathrm{GHz}$ of Fig. 9(b) is seen to be rapidly neutralized by simply increasing the slab permittivity of the RPA, even just mildly. For this case, the best $90 \%$ performance of Fig. 10(a) is equaled in Fig. 11, and this is not to mention that the profile of the RPA cover remains lower than any of those aforementioned plain sheets.

Observing Fig. 10(a) and (b) again, a fairly high $80 \%$ efficiency of the thin sheet $(d=1.13 \mathrm{~mm})$ with high $\varepsilon_{r}=9$ exceedingly surpasses the $53 \%$ attainable by the corresponding uncovered FPA at that same 5-GHz frequency. Hence, thin boards with high permittivities are indeed effective tuners at low frequencies as theorized. However, what about their bandwidths, which are known to be an issue for slabs with high $\varepsilon_{r}$ values? We will look into this next.

The same $80 \%$ level of performance at $5 \mathrm{GHz}$ can be attained by using an RPA cover with $\varepsilon_{r}=2.2, d=1.13 \mathrm{~mm}$, and patch-to-cell-size ratio of 0.884 (a large patch), as evident from Fig. 9(b). Hence, with these two aforementioned matching structures having the same $80 \%$ coupling efficiency at $5 \mathrm{GHz}$, namely: 1) plain sheet with $\varepsilon_{r}=9, d=1.13 \mathrm{~mm}$, and 2) RPA cover with $\varepsilon_{r}=2.2, d=1.13 \mathrm{~mm}$, and patch-to-cell-size ratio $=0.884$, a matching bandwidth comparison study between them will now be conducted. Fig. 12 shows this comparison, in which the coupling efficiency of the uncovered FPA is included. Instead of ideal HRW elements as in Figs. 9(b) and 10, realistic waveguide elements with fixed dielectric sidewall loadings of 1-mm thickness and hard only at the center 5-GHz frequency are now used in this bandwidth study of Fig. 12(a), over the range from 4.96 to $5.1 \mathrm{GHz}$. As can be seen, the matching performance of the RPA cover exceeds that of the plain sheath for frequencies above $5.02 \mathrm{GHz}$. Hence, the bandwidths of both types of covers are comparable.

It is well known that the bandwidth can be increased by increasing the slab thickness and lowering the permittivity. Hence, giving the plain sheets this advantage, further comparisons are made in the same Fig. 12(a) for two other plain sheets with lower permittivities and larger thicknesses, both attaining that same $80 \%$ at $5 \mathrm{GHz}$. The parameters of these two plain sheets are: 1) $\varepsilon_{r}=6, d=4.5 \mathrm{~mm}$, and 2) $\varepsilon_{r}=3, d=6.75 \mathrm{~mm}$, as deducible from Fig. 10(a) or (b). Likewise, realistic waveguide elements are considered here. As can be observed from the other traces in Fig. 12(a), the RPA cover is still superior at frequencies below $5.03 \mathrm{GHz}$, but loses out to the two contending plain sheets only at higher frequencies. Hence, depending on whether higher or lower frequency bands are more important, there is no clear cut bandwidth improvement of the plain sheets over the RPA cover (despite the advantages granted to the former), at least over the investigated frequency range of Fig. 12. Hence, the bandwidth advantage of the RPA cover over the plain sheath is found to be resilient.

As supplementary studies, Fig. 12(b) shows another bandwidth study over the same frequency range, comparing the same RPA cover of Fig. 12(a) with two other plain sheets which attain a higher $90 \%$ radiation level at $5 \mathrm{GHz}$ (as conveyed by Fig. 10). Hence, the sheets are again given an edge over the RPA covers. The parameters of these two sheaths are: 1) $\varepsilon_{r}=7, d=3.375$ $\mathrm{mm}$, and 2) $\varepsilon_{r}=5, d=4.5 \mathrm{~mm}$. This time, however, the advantage granted to the plain sheets is too strong for the RPA cover to overcome, as it succumbs to the sheaths in bandwidth performance. Nonetheless, to be subdued by an unfair $10 \%$ efficiency advantage is still honorable.

Moreover, it is observed from Fig. 12(a) and (b) that the RPA cover is able to enhance the efficiency of its uncovered array by $25 \%-30 \%$ over the entire band. Therefore, the ability of the RPA cover to significantly improve the radiation of uncovered FPAs over a decent bandwidth is demonstrated.

In conclusion of the findings made in this section, where low-frequency matching is concerned, the RPA cover with a thin board of low permittivity and printed by large patches is superior to 1) thick boards of that same low permittivity - in terms of profile and weight advantages, as well as maximum attainable radiation efficiency, and 2) boards with the same thickness as of the RPA cover, but with high permittivities-in terms of bandwidth resilience. 


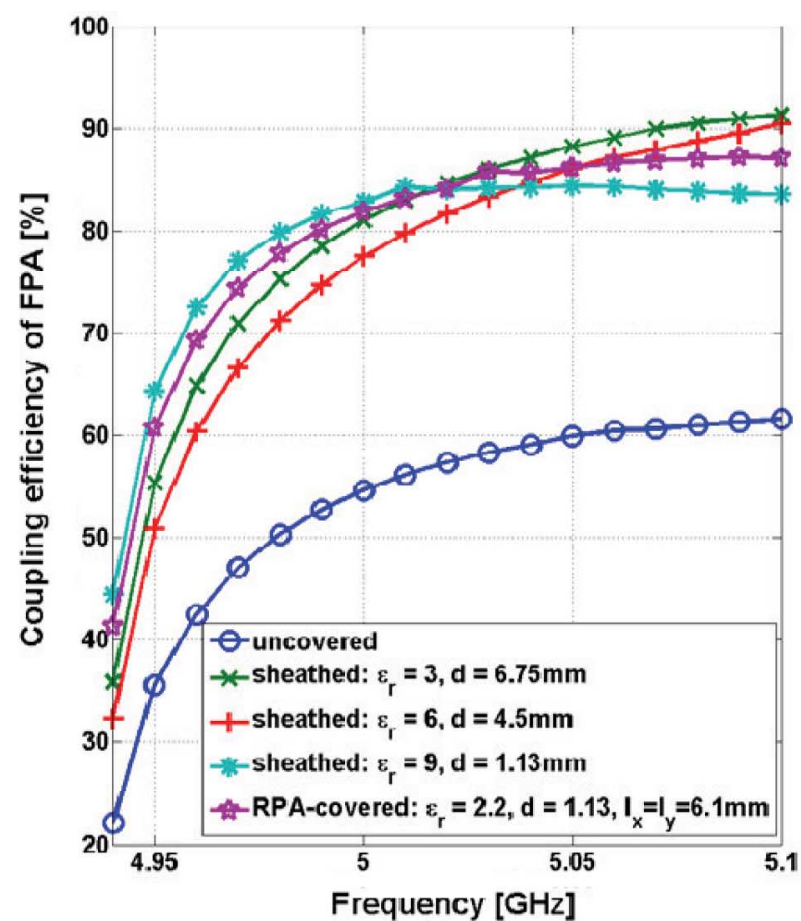

(a)

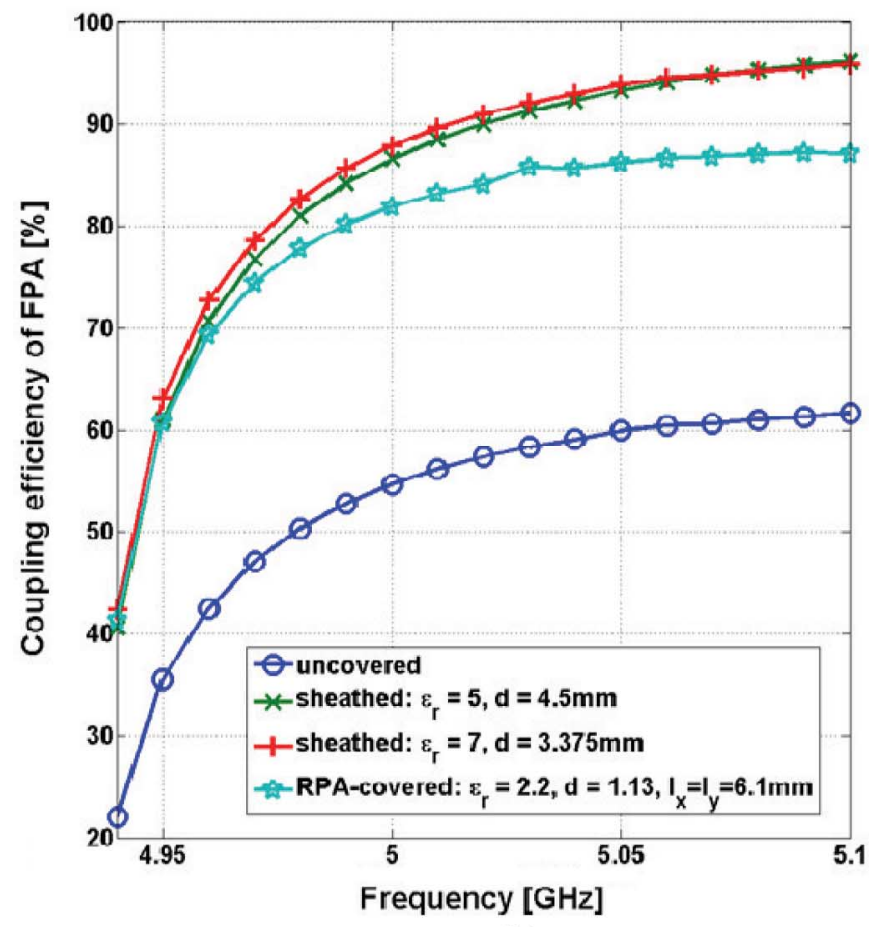

(b)

Fig. 12. Bandwidth study about $5 \mathrm{GHz}$, for realizable (nonideal) HRW FPA: comparison between the best performing RPA cover of Fig. 9(b) and (a) three plain-sheet cases of Fig. 10 attaining the same $80 \%$ performance level: $\varepsilon_{r}=$ $3, d=6.75 \mathrm{~mm}, \varepsilon_{r}=6, d=4.5 \mathrm{~mm}$, and $\varepsilon_{r}=9, d=1.13 \mathrm{~mm}$; and t(bo) other plain-sheet cases of Fig. 10 , but attaining a higher $\approx 90 \%$ radiation: $\varepsilon_{r}=7, d=3.375 \mathrm{~mm}$, and $\varepsilon_{r}=5, d=4.5 \mathrm{~mm}$. For all cases, the sidewall dielectric thickness of the waveguide element is maintained at $1 \mathrm{~mm}$, and it is hard only at $5 \mathrm{GHz}$.

\section{CONCLUSION}

This work has studied an HRW array covered by a plain sheath and an RPA cover. The prospects of such layers in im- proving the radiation efficiency of FPAs as reflector feeds were investigated. When the profile, weight, and bandwidth of the covers do not matter, the matching capabilities of both matching layers have been found to be comparable. The feasibility of the RPA cover as a new type of effective array match tuner is thus established. It is also shown to be a viable alternative to conventional plain sheath tuners. The parameters of both forms of matching layers can be tuned to achieve either low-frequency albeit narrowband matching, or broadband matching but with reduced low-frequency matching effects. This work has ascertained how this can be done. A tradeoff between broadband and low-frequency matching, however, must be made.

However, when the profile, weight, and bandwidth of the matching structure do matter, the advantages of the RPA cover over the plain dielectric sheet are clearly demonstrated in this paper. The use of a thin slab of low permittivity but printed with an array of large metal patches (relative to the unit cell) has proved to be a superior matching structure as compared to either a thick plain dielectric sheet of that same low permittivity in terms of profile and weight, or a thin sheet of high permittivity in terms of bandwidth.

In view of today's advanced printed-circuit-board (PCB) technology allowing for fast and low cost production of microstrip patch arrays, the ability to tune the matching capabilities of the RPA layer by appropriately adjusting the metal patch parameters provides a significant advantage over the tuning parameters of plain dielectric sheaths. Hence, rather than tuning by changing the permittivities and thicknesses of plain sheaths, being often a time-consuming and expensive procedure (requiring the reacquisition or remachining of the slabs), the tuning by modifications of patch parameters permits the use of a fixed dielectric board, thus offering new dimensions of speed, cost savings, and convenience. Note that in using RPA covers, the dielectric board is kept only as a support, over which the patch array is etched. Its presence is not necessary for matching and can be made extremely thin.

It has been shown that both forms of matching layers (sheath and RPA layer) can be tuned to achieve excellent low-frequency matching. This thereby responds well to the problems of radiation deficiency suffered by arrays at low frequencies arising from increased mutual coupling losses among the elements. This paper has carried out foundational investigations into the topic of FPA matching, and it is hoped that the findings here are able to galvanize future studies on this challenging and important subject. Further work could be to optimize the parameters of the waveguide array and the RPA structure such that the frequency band in which the coupling efficiency is maintained above a certain threshold value is either maximized or tuned. Different shapes of the metallization can also be investigated.

\section{REFERENCES}

[1] T. S. Bird, "A multibeam feed for the Parkes radio telescope," in Proc. IEEE Antennas Propag. Soc. Int. Symp., Jun. 1994, pp. 966-969.

[2] B. Saka and E. Yazgan, "Pattern optimization of a reflector antenna with planar-array feeds and cluster feeds," IEEE Trans. Antennas Propag., vol. 45, no. 1, pp. 93-97, Jan. 1997.

[3] T. S. Bird and G. Cortés-Medellín, "Multibeam feed array design for the Arecibo radio telescope," in Proc. IEEE Antennas Propag. Soc. Int. Symp., Jun. 2003, vol. 1, pp. 116-119. 
[4] A. K. Bhattacharyya and G. Goyette, "A novel horn radiator with high aperture efficiency and low cross-polarization and applications in arrays and multibeam reflector antennas," IEEE Trans. Antennas Propag., vol. 52, no. 11, pp. 2850-2859, Nov. 2004.

[5] K. K. Chan and S. K. Rao, "Design of high efficiency circular horn feeds for multibeam reflector applications," IEEE Trans. Antennas Propag., vol. 56, no. 1, pp. 253-258, Jan. 2008.

[6] M. V. Ivashina, J. D. Bregman, and A. van Ardenne, "A way to improve the field of view of the radio-telescope with a dense focal plane array," in Proc. 12th Int. Crimean Conf. Microw. Telecommun. Technol., Sevastopol, Crimea, Ukraine, Sep. 2002, pp. 278-281.

[7] M. V. Ivashina, J. D. Bregman, J. G. Bij de Vaate, L. Li, and A. J. Parfitt, "Experimental results for a focal plane array synthesized with conjugate field method," in Proc. IEEE Antennas Propag. Soc. Int. Symp., 2004, vol. 1, pp. 21-24.

[8] P. Hannan, D. Lerner, and G. Knittel, "Impedance matching a phasedarray antenna over wide scan angles by connecting circuits," IEEE Trans. Antennas Propag., vol. AP-13, no. 1, pp. 28-34, Jan. 1965.

[9] W. K. Kahn, "Impedance-match and element-pattern constraints for finite arrays," IEEE Trans. Antennas Propag., vol. AP-25, no. 6, pp. 747-755, Nov. 1977

[10] M. Ng Mou Kehn and P.-S. Kildal, "Miniaturized rectangular hard waveguides for use in multifrequency phased arrays," IEEE Trans. Antennas Propag., vol. 53, no. 1, pp. 100-109, Jan. 2005.

[11] J. J. Lee and R. Chu, "Aperture matching of a dielectric loaded circular waveguide element array," IEEE Trans. Antennas Propag., vol. 37, no. 3, pp. 395-399, Mar. 1989.

[12] E. G. Magill and H. A. Wheeler, "Wide-angle impedance matching of a planar array antenna by a dielectric sheet," IEEE Trans. Antennas Propag., vol. 14, no. 1, pp. 49-53, Jan. 1966.

[13] H. Holter, "Dual-polarized broadband array antenna with BOR-elements, mechanical design and measurements," IEEE Trans. Antennas Propag., vol. 55, no. 2, pp. 305-312, Feb. 2007.

[14] M. Ng Mou Kehn, M. V. Ivashina, P.-S. Kildal, and R. Maaskant, "Definition of unifying decoupling efficiency of different array antennas-Case study of dense focal plane array feed for parabolic reflector," Int. J. Electron. Commun., 2009, doi: 10.1016/j.aeue. 2009.02.011.

[15] M. V. Ivashina, M. Ng Mou Kehn, P.-S. Kildal, and R. Maaskant, "Decoupling efficiency of a wideband Vivaldi focal plane array feeding a reflector antenna," IEEE Trans. Antennas Propag., vol. 57, no. 2, pp. 373-382, Feb. 2009.

[16] A. K. Bhattacharyya, Phased Array Antennas - Floquet Analysis, Synthesis, BFNs, and Active Array Systems. Hoboken, NJ: Wiley, 2006.

[17] S. M. Rao, D. R. Wilton, and A. W. Glisson, "Electromagnetic scattering by surfaces of arbitrary shape," IEEE Trans. Antennas Propag., vol. AP-30, no. 3, pp. 409-418, May 1982.

[18] Z. Sipus, P.-S. Kildal, R. Leijon, and M. Johansson, "An algorithm for calculating Green's functions of planar, circular cylindrical, and spherical multilayer structures," Appl. Comput. Electromagn. Soc. J., vol. 13 , no. 3, pp. 243-254, Nov. 1998.

[19] M. Ng Mou Kehn, M. Nannetti, A. Cucini, S. Maci, and P.-S. Kildal, "Analysis of dispersion in dipole-FSS loaded hard rectangular waveguide," IEEE Trans. Antennas Propag., vol. AP-54, no. 8, pp. 2275-2282, Aug. 2006.

[20] M. Ng Mou Kehn and L. Shafai, "Characterization of dense focal plane array feeds for parabolic reflectors in achieving closely-overlapping or widely-separated multiple beams," Radio Sci., Jun. 25, 2008, doi: 10.1029/2008RS003953.

[21] N. Amitay, V. Galindo, and C. P. Wu, Theory and Analysis of Phased Array Antennas. New York: Wiley, 1972.

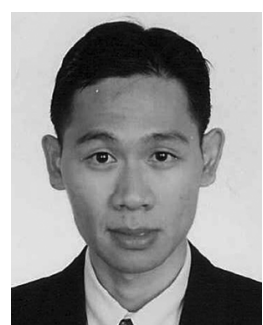

Malcolm Ng Mou Kehn (S'02-M'06) was born in Singapore on September 26, 1976. He received the B.Eng. (honors) degree from the National University of Singapore, Singapore, in 2001, and the Licentiate and $\mathrm{Ph} . \mathrm{D}$. degrees from Chalmers University of Technology, Gothenburg, Sweden, in 2004 and 2005, respectively, all in electrical engineering.

During 2006-2008, he was a Postdoctoral Fellow at the Department of Electrical and Computer Engineering, University of Manitoba, Winnipeg, MB, Canada. Following this, he proceeded to Concordia
University, Montreal, QC, Canada, for a year. Currently, he is with the Department of Electrical Engineering, National Chiao Tung University, Hsinchu, Taiwan, serving as an Assistant Professor. He had been actively involved in research projects funded by the Swedish Defense Research Agency between 2002 and 2006. During autumn 2004, he spent several months at the University of Siena, Italy, for a research visit. In December 2004, he visited the University of Zagreb, Croatia, as an invited speaker where he gave an IEEE lecture, in connection with the IEEE Croatia Chapter activities.

Dr. Ng Mou Kehn received the Union Radio-Scientifique Internationale (URSI) Young Scientist Award in 2007

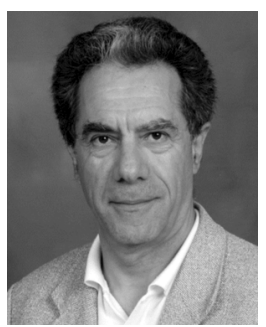

Lotfollah Shafai (S'67-M'69-SM'75-F'88-LF'07) received the B.Sc. degree from the University of Tehran, Tehran, Iran, in 1963 and the M.Sc. and $\mathrm{Ph} . \mathrm{D}$. degrees from the Faculty of Applied Sciences and Engineering, University of Toronto, Toronto, ON, Canada, in 1966 and 1969, all in electrical engineering.

In November 1969, he joined the Department of Electrical and Computer Engineering, University of Manitoba, Winnipeg, MB, Canada, as a Sessional Lecturer. He became an Assistant Professor in 1970, an Associate Professor in 1973, and the Professor in 1979. Since 1975, he has made special effort to link the University research to the industrial development, by assisting industries in the development of new products or establishing new technologies. To enhance the University of Manitoba contact with industry, in 1985 he assisted in establishing The Institute for Technology Development, and was its Director until 1987, when he became the Head of the Electrical Engineering Department. His assistance to industry was instrumental in establishing an Industrial Research Chair in Applied Electromagnetics at the University of Manitoba in 1989, which he held until July 1994.

Dr. Shafai has been a participant in nearly all Antennas and Propagation symposia and participates in the review committees. He is a member of Union Radio Scientifique Internationale (URSI) Commission B and was its Chairman during 1985-1988. In 1986, he established the Symposium on Antenna Technology and Applied Electromagnetics (ANTEM) at the University of Manitoba that is currently held every two years. He has been the recipient of numerous awards. In 1978, his contribution to the design of a small ground station for the Hermus satellite was selected as the 3rd Meritorious Industrial Design. In 1984, he received the Professional Engineers Merit Award, and in 1985, "The Thinker" Award from Canadian Patents and Development Corporation. From the University of Manitoba, he received the "Research Awards" in 1983, 1987, and 1989, the Outreach Award in 1987, and the Sigma Xi, Senior Scientist Award in 1989. In 1990, he received the Maxwell Premium Award from IEE (London), and in 1993 and 1994, the Distinguished Achievement Awards from Corporate Higher Education Forum. In 1998, he received the Winnipeg RH Institute Foundation Medal for Excellence in Research. In 1999 and 2000, he received the University of Manitoba, Faculty Association Research Award. He was elected a Fellow of The Royal Society of Canada in 1998. He was a recipient of the IEEE Third Millennium Medal in 2000, and in 2002, was elected a Fellow of The Canadian Academy of Engineering and Distinguished Professor at The University of Manitoba. In 2003, he received the IEEE Canada "Reginald A. Fessenden Medal" for "Outstanding Contributions to Telecommunications and Satellite Communications," and Natural Sciences and Engineering Research Council (NSERC) Synergy Award for "Development of Advanced Satellite and Wireless Antennas." In 2009, he was elected a Fellow of the Engineering Institute of Canada, and was the recipient of the IEEE Chen-To-Tai Distinguished Educator Award. He holds a Canada Research Chair in Applied Electromagnetics and was the International Chair of Commission B of International Union of Radio Science (URSI) for 2005-2008. 